https://doi.org/10.15407/ujpe63.5.431

V.O. GUBANOV, A.P. NAUMENKO, M.M. BILIY, I.S. DOTSENKO, O.M. NAVOZENKO, M.M. SABOV, L.A. BULAVIN

Taras Shevchenko National University of Kyiv

(64/13, Volodymyrs'ka Str., Kyiv 01601, Ukraine)

\title{
ENERGY SPECTRA CORRELATION OF VIBRATIONAL AND ELECTRONIC EXCITATIONS AND THEIR DISPERSION IN GRAPHITE AND GRAPHENE
}

The correlation between the vibrational and electron excitation modes in the energy spectra of single-layer graphene and crystalline graphite, as well as the dispersion dependences of those modes, has been studied. The methods of the theory of projective representations of the point and spatial symmetry groups are used for the first time in order to interpret those correlations. The correlations of vibrational and electron excitation spectra and the compatibility conditions for irreducible projective representations in the descriptions of quantum states of graphene and crystalline graphite at various points of their Brillouin zones are determined. For the projective representations of all projective classes belonging to the hexagonal system, standard factor-systems are constructed for the first time. In particular, the factor-systems for electron states are first determined. The results obtained are used to calculate, also for the first time, the correct spinor multiplication tables, i.e. the multiplication tables for elements in double symmetry groups. The developed method is applied to classify all high-symmetry points in the Brillouin zones of single-layer graphene and crystalline graphite with respect to the symmetry type of vibrational excitations.

Ke ywords: spinor representation of symmetry groups, factor-system, dispersion of elementary excitations.

\section{Introduction}

The study of the energy spectra of collective vibrational and electron excitations in crystals and oneand biperiodic nanostructures, as well as their dispersion, always attracted large attention of the scientific community, because such researches are classified as fundamental and undoubtedly possess both practical and theoretical values. Nevertheless, today, there is neither a complete understanding of the dispersion of collective excitations nor their theoretical or symmetric interpretation, even for such a widely researched structure as crystalline graphite. The classes of projective representations, according to which the wave functions of vibrational and electron states at various points of the Brillouin zone in graphite are transformed, remain unknown. The changes in the degeneration character of the energy spectrum of excitations and their correlation with the respective excita-

(C) V.O. GUBANOV, A.P. NAUMENKO, M.M. BILIY, I.S. DOTSENKO, O.M. NAVOZENKO, M.M. SABOV, L.A. BULAVIN, 2018 tions in single-layer graphene are not determined. At present, there are no clear ideas concerning a correct construction of factor-systems belonging to different projective classes, as well as their reduction to the standard form, which determines the shape of dispersion curves and the energy state degeneration multiplicity.

In this paper, the methods have been developed for determining the projective classes themselves and their changes at the Brillouin zone boundaries, as well as the irreducible projective representations, according to which the wave functions of the researched quantum-mechanical systems are transformed. For the first time, the methods for constructing factorsystems that characterize a certain projective class and their reduction to the standard form are presented, a new classification of projective classes for hexagonal structures is proposed, and a correct table for the symmetry transformations of the wave functions of quantum states with a half-integer spin, i.e. spinors, is determined. The symmetry analysis of the dispersion of vibrational and electron excitations 


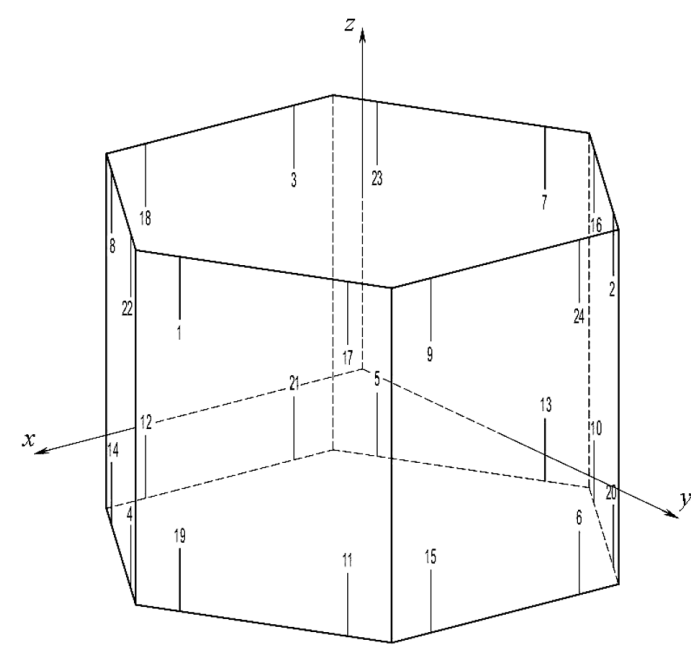

Fig. 1. Prism of reindicated $H$-rotations

is first carried out for the $\pi$-bands of graphite crystals, and their correlations with the electron $\pi$-bands in single-layer graphene are discussed.

\section{System of H-Rotations of the Group 6/mmm $\left(D_{6 h}\right)$}

Similarly to works $[1,2]$, we introduce a right-handed oblique coordinate system with the axis $Z$ directed upward and the axis $Y$ directed to the right (the angle between the $X$ and $Y$ axes equals $120^{\circ}$ ) for $\mathrm{H}$ rotations (the rotational elements of both the first and second kinds) of the group $6 / \mathrm{mmm}\left(D_{6 h}\right)$. We also use the digital notation for the elements, but their ordinal numbers are somewhat changed. This change is made for a more convenient representation of the "block" character of the tables. This character is determined by the internal structure of the group $6 / m m m\left(D_{6 h}\right)$, namely, by the fact that the group $D_{6 h}$ is a direct product of the groups $D_{6}$ and $C_{i}$, i.e. $D_{6 h}=D_{6} \times C_{i}$, and the group $D_{6}$, in turn, is a direct product of groups $D_{3}$ and $C_{2}$, i.e. $D_{6}=D_{3} \times C_{2}$. The group $D_{3}$ contains an invariant subgroup, which can be used to construct its factor group, the latter being isomorphic to the group $C_{2}$. According to this "composition principle", the "principal" axis that determines symbols in the notation of irreducible representations of the group $D_{6 h}$ is the element $c_{3}$, i.e. the rotation around the third-order axis in the higher-rank subgroup in the case where the highest-rank subgroup is the first in all expressions for direct products. The meaning of H-rotations is explained in Fig. 1. As was done in works $[1,2]$, the inversion rotations have a priority over the mirror ones.

\section{Crystal Structures, Brillouin Zones, and Basic Symmetry Elements}

Figure 2, a demonstrates a standard unit cell in the crystal lattice of Bernal graphite $\gamma-C$ [3]. The corresponding standard diagram of its spatial symmetry group $P 6_{3} / m m c\left(D_{6 h}^{4}\right)$ is shown in Fig. 2, b. Figure 2, $c$ illustrates the position and orientation of symmetry elements of the point group $6 / \mathrm{mmm}\left(D_{6 h}\right)$.

Figure 3 exhibits the Brillouin zone of the $\gamma-C$ graphite crystals and its symmetry points. The latter are marked by letters in Herring's notation for hexagonal structures [4]. The Wigner-Seitz unit cell and the Brillouin zone for single-layer graphene $C_{L 1}$ are demonstrated in Figs. 4, $a$ and 4, b, respectively.

In Fig. 4, $a$, the solid lines are used to schematically mark a unit cell of graphene $C_{L 1}$, show the primitive translation vectors $\mathbf{a}_{1}$ and $\mathbf{a}_{2}$, and indicate the orientation of graphene symmetry elements in the threedimensional space that was used in calculations. The dashed lines illustrate the corresponding orientations of the reciprocal lattice vectors $\mathbf{b}_{1}$ and $\mathbf{b}_{2}$ on an arbitrary scale and the positions of reciprocal lattice sites in the reciprocal space. In Fig. $4, b$, on the contrary, the solid lines are used to show the reciprocal lattice vectors, and the dashed ones to demonstrate the direct lattice vectors. The unit cells (the WignerSeitz cells) of the graphene layer in the coordinate (Fig. 4, a) and reciprocal (Fig. 4, b) spaces (in the latter case, this cell coincides with the first Brillouin zone) are tinted grey. It is of interest that those cells are rotated by an angle of $\pi / 2$ with respect to each other (or by an angle of $30^{\circ}$, taking into account that the rotation by $60^{\circ}$ is a symmetry element in the both cases). In Fig. 4, $b$, the high-symmetry points $K$ and $M$ in the Brillouin zone of graphene are also indicated. The equivalent points are marked by one or two primes.

The spatial symmetry group of the crystalline graphite lattice, $P 6_{3} / m m c\left(D_{6 h}^{4}\right)$, is non-symmorphic. It is determined by the basic (main) elements, which can be chosen as follows:

$h_{1}=(0 \mid e), h_{2}=\left(0 \mid c_{3}\right), h_{3}=\left(0 \mid c_{3}^{2}\right), h_{4}=\left(0 \mid\left(u_{2}\right)_{1}\right)$, $h_{5}=\left(0 \mid\left(u_{2}\right)_{2}\right), h_{6}=\left(0 \mid\left(u_{2}\right)_{3}\right), h_{7}=\left(\frac{\mathbf{a}_{\mathbf{1}}}{2} \mid c_{2}\right)$,

$h_{8}=\left(\frac{\mathbf{a}_{\mathbf{1}}}{2} \mid c_{6}^{5}\right), h_{9}=\left(\frac{\mathbf{a}_{\mathbf{1}}}{2} \mid c_{6}\right), h_{10}=\left(\frac{\mathbf{a}_{\mathbf{1}}}{2} \mid\left(u_{2}^{\prime}\right)_{1}\right)$,

ISSN 2071-0186. Ukr. J. Phys. 2018. Vol. 63, No. 5 

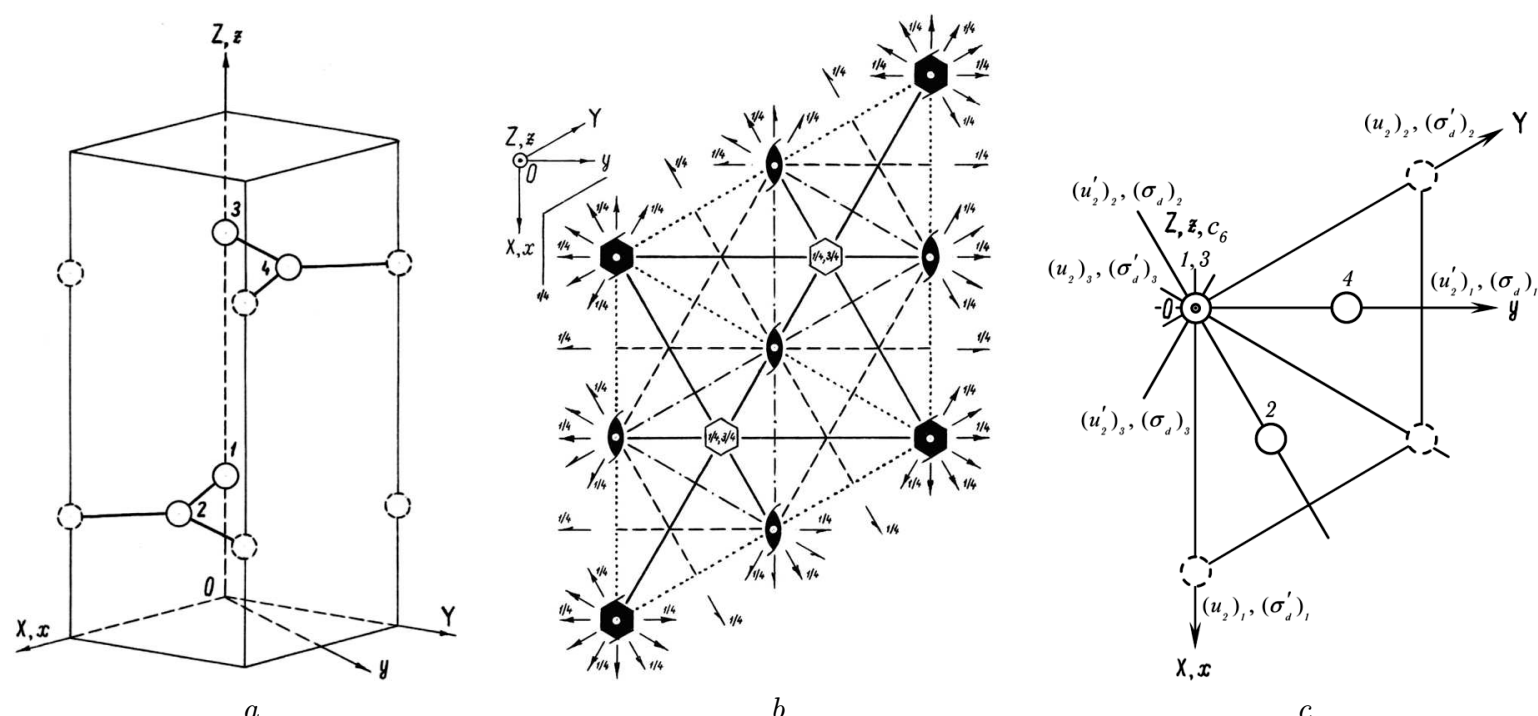

Fig. 2. Structure of the standard unit cell of crystalline graphite $\gamma-C(a)$, the standard diagram of the spatial symmetry group $P 6_{3} / m m c\left(D_{6 h}^{4}\right)(b)$. arrangement and orientation of the elements of the point symmetry group $6 / m m m\left(D_{6 h}\right)$ (c). The circles indicate the positions of carbon atoms

$$
\begin{aligned}
& h_{11}=\left(\frac{\mathbf{a}_{\mathbf{1}}}{2} \mid\left(u_{2}^{\prime}\right)_{2}\right), h_{12}=\left(\frac{\mathbf{a}_{\mathbf{1}}}{2} \mid\left(u_{2}^{\prime}\right)_{3}\right), h_{13}=(0 \mid i), \\
& h_{14}=\left(0 \mid i c_{3}\right), h_{15}=\left(0 \mid i c_{3}^{2}\right), h_{16}=\left(0 \mid i\left(u_{2}\right)_{1}\right), \\
& h_{17}=\left(0 \mid i\left(u_{2}\right)_{2}\right), h_{18}=\left(0 \mid i\left(u_{2}\right)_{3}\right), h_{19}=\left(\frac{\mathbf{a}_{\mathbf{1}}}{2} \mid i c_{2}\right), \\
& h_{20}=\left(\frac{\mathbf{a}_{\mathbf{1}}}{2} \mid i c_{6}^{5}\right), h_{21}=\left(\frac{\mathbf{a}_{\mathbf{1}}}{2} \mid i c_{6}\right), h_{22}=\left(\frac{\mathbf{a}_{\mathbf{1}}}{2} \mid i\left(u_{2}^{\prime}\right)_{1}\right), \\
& h_{23}=\left(\frac{\mathbf{a}_{\mathbf{1}}}{2} \mid i\left(u_{2}^{\prime}\right)_{2}\right), h_{24}=\left(\frac{\mathbf{a}_{\mathbf{1}}}{2} \mid\left(u_{2}^{\prime}\right)_{3}\right),
\end{aligned}
$$

where $\mathbf{a}_{1}$ is the primitive vector of the crystal lattice directed along the axis $O Z(O z)$. At the same time, the spatial symmetry group of the crystalline lattice of single-layer graphene, $P 6 / \mathrm{mmm}(D G 80)[5]$ or the triperiodic group $D_{6 h}^{1}$, whose diagram coincides with that of the biperiodic spatial group of single-layer graphene, is symmorphic, and all its "rotational" elements - the symmetry elements of the point group $6 / m m m\left(D_{6 h}\right)-$ do not contain nontrivial (partial) translations.

\section{New Classification of Projective Classes for Projective Representations of Group 6/mmm $\left(D_{6 h}\right)$ and Construction of Corresponding Standard Factor-Systems}

The determination of the representations $D_{\{\mathbf{k}\}}$, i.e. the irreducible representations of the spatial

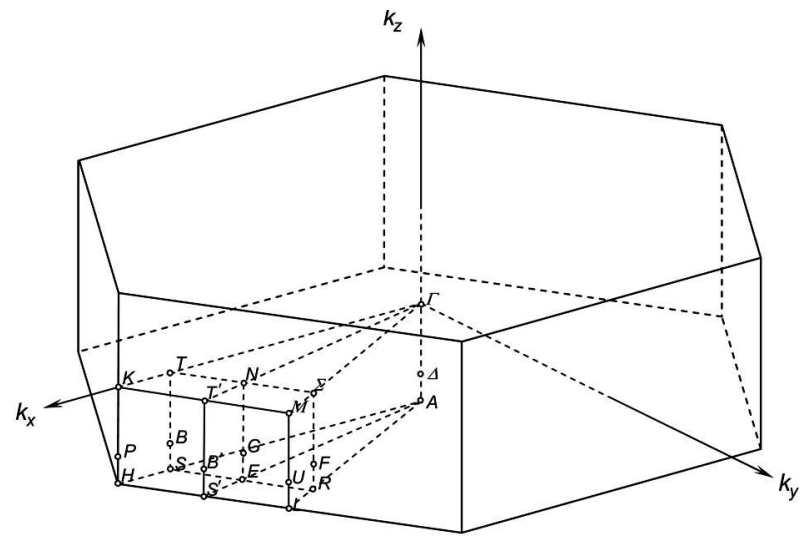

Fig. 3. Brillouin zone of $\gamma-C$ graphite crystals and its symmetry points

group $G$ with an irreducible star $\{\mathbf{k}\}$ of the wave vector $\mathbf{k}$, is reduced to the finding of projective representations for the point group of equivalent directions $F_{\mathbf{k}}$ of the wave-vector group $G_{\mathbf{k}}$, the subgroup of group $G$. The groups $F_{\mathbf{k}}$ are possible subgroups of the point symmetry group of equivalent directions $F$, which characterizes the crystalline class of the crystal lattice. A general method to construct irreducible representations of the wave-vector group $D_{\mathbf{k}}$, which are also called small representations, in the form of projective representations of the point groups of equivalent directions of the wave-vector groups $F_{\mathbf{k}}$, which 

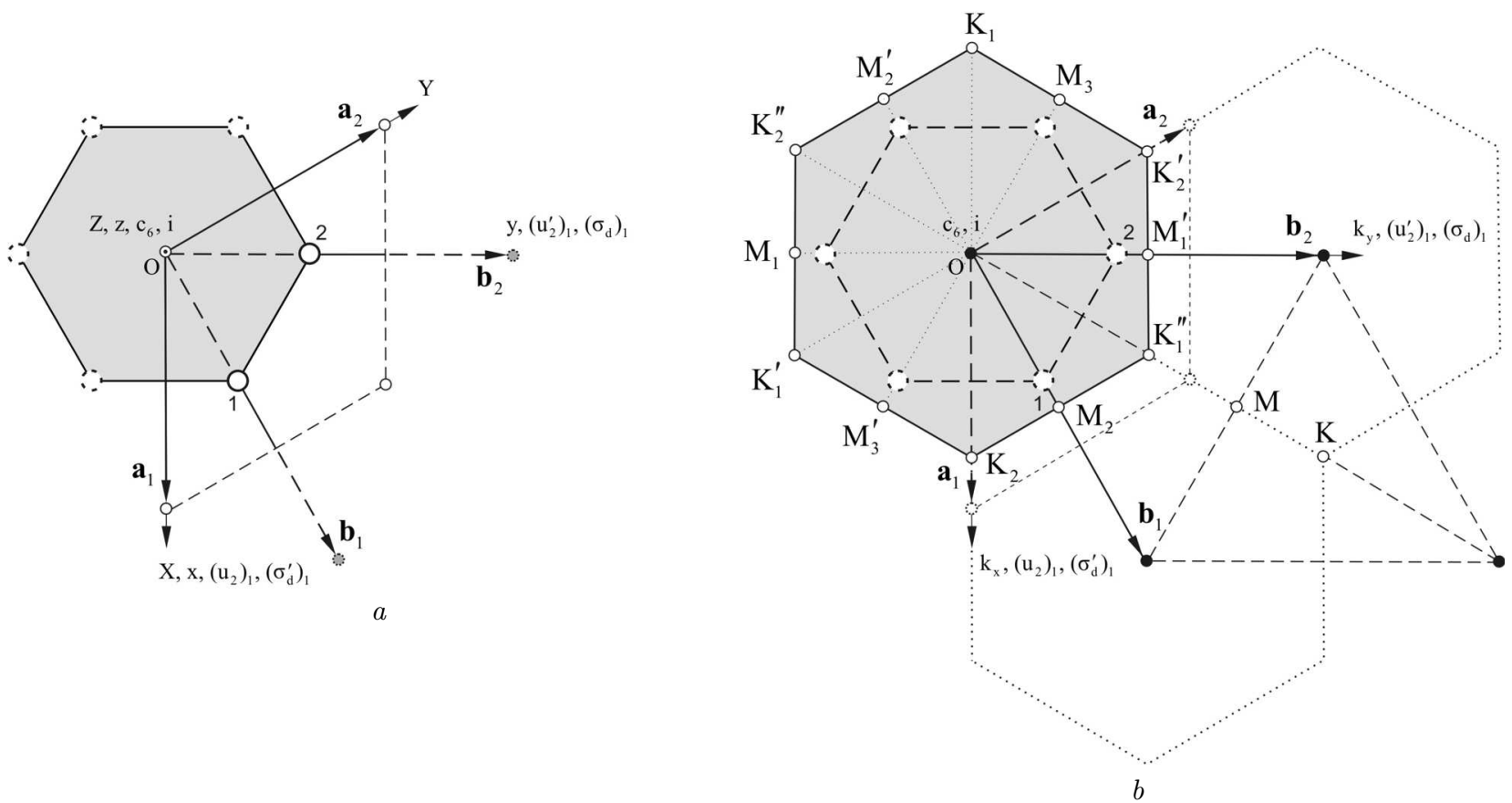

Fig. 4. Wigner-Seitz unit cells $(a)$ and Brillouin zone of single-layer graphene $C_{L 1}(b)$

are isomorphic to the factor groups of the group $G_{\mathbf{k}}$ with respect to the infinite translation-invariant subgroup, is described in work [6].

We recall that the projective or ray representations are those representations that satisfy the relation

$D\left(r_{2}\right) D\left(r_{1}\right)=\omega\left(r_{2}, r_{1}\right) D\left(r_{2} r_{1}\right)$,

where $r_{i}$ is a "rotational" (of the first or second kind) element of the group $F_{\mathbf{k}}$, and $\omega\left(r_{2}, r_{1}\right)$ is a set of $h^{2}$ complex, in the general case, numerical coefficients $(h$ is the order of group), which is called a factor-system and has the following properties:

$\left|\omega\left(r_{2}, r_{1}\right)\right|=1$

and

$\omega\left(r_{3}, r_{2} r_{1}\right) \omega\left(r_{2}, r_{1}\right)=\omega\left(r_{3}, r_{2}\right) \omega\left(r_{3} r_{2}, r_{1}\right)$.

If $D(r)$ is a certain projective representation belonging to the factor-system $\omega\left(r_{2}, r_{1}\right)$, then any other projective representation

$D^{\prime}(r)=\frac{D(r)}{u(r)}$,

where $u(r)$ is an arbitrary single-valued function on the group $L$ and $|u(r)|=1$, is also a projective rep- resentation of the group $L$, but with another factorsystem $\omega^{\prime}\left(r_{2}, r_{1}\right)$, i.e.

$D^{\prime}\left(r_{2}\right) D^{\prime}\left(r_{1}\right)=\omega^{\prime}\left(r_{2}, r_{1}\right) D^{\prime}\left(r_{2} r_{1}\right)$,

where

$\omega^{\prime}\left(r_{2}, r_{1}\right)=\frac{\omega\left(r_{2}, r_{1}\right) u\left(r_{2} r_{1}\right)}{u\left(r_{1}\right) u\left(r_{2}\right)}$.

All factor-systems related by expression (5) are called projective-equivalent (or $p$-equivalent). A set of all $p$ equivalent factor-systems is called the class of factorsystems. This is also true for the corresponding projective representations.

The group $6 / \mathrm{mmm}$ has eight classes of projective representations. They are classified by means of a system of three coefficients $-\alpha, \beta$, and $\gamma-$ which take a value of 1 or -1 . Each coefficient is determined by the relation

$\left[\omega\left(r_{j}, r_{i}\right)\right]^{-1} \omega\left(r_{i}, r_{j}\right)=\frac{\omega\left(r_{i}, r_{j}\right)}{\omega\left(r_{j}, r_{i}\right)}$

for the corresponding pair of commuting elements $r_{i}$ and $r_{j}$ selected from the group generators or the elements belonging to different classes containing the generators. As commuting elements for the group $6 / \mathrm{mmm}$, it is convenient to choose, e.g., the following

ISSN 2071-0186. Ukr. J. Phys. 2018. Vol. 63, No. 5 
group generators: $a=r_{1}=\left(u_{2}\right)_{1}, b=r_{2}=c_{2}$, and $c=r_{3}=i$. In this case,

$\alpha=\frac{\omega(a, b)}{\omega(b, a)}=\frac{\omega\left[\left(u_{2}\right)_{1}, c_{2}\right]}{\omega\left[c_{2},\left(u_{2}\right)_{1}\right]}$,

$\beta=\frac{\omega(a, c)}{\omega(c, a)}=\frac{\omega\left[\left(u_{2}\right)_{1}, i\right]}{\omega\left[i,\left(u_{2}\right)_{1}\right]}$,

$\gamma=\frac{\omega(b, c)}{\omega(c, b)}=\frac{\omega\left(c_{2}, i\right)}{\omega\left(i, c_{2}\right)}$.

For the classification and notation of the factorsystem classes and, respectively, the classes of projective representations in the group $6 / \mathrm{mmm}$, it is convenient to choose the following system:

- $\operatorname{class} K_{0}: \alpha=1, \beta=1, \gamma=1$;

- class $K_{1}: \alpha=-1, \beta=1, \gamma=1$;

- class $K_{2}: \alpha=1, \beta=-1, \gamma=1$;

- class $K_{3}: \alpha=-1, \beta=-1, \gamma=1$;

- class $K_{4}: \alpha=1, \beta=1, \gamma=-1$;

- class $K_{5}: \alpha=-1, \beta=1, \gamma=-1$;

- class $K_{6}: \alpha=1, \beta=-1, \gamma=-1$;

- $K_{7}: \alpha=-1, \beta=-1, \gamma=-1$.

This system is substantially different from that proposed in work [6] by both the definitions of the coefficients and their values.

The irreducible representations of the wave-vector group $D_{\mathbf{k}}$ contain an infinite number of members $D_{\mathbf{k}}(h)$ for the elements $h \in G_{\mathbf{k}}$. Every element $h$ can be expressed in the form $h=(\boldsymbol{\alpha}+\mathbf{a} \mid r)$, where $r$ is a "rotational" element from the set forming the group $F_{\mathbf{k}}, \boldsymbol{\alpha}$ is the vector of a nontrivial translation corresponding to the rotational element $r$, and $\mathbf{a}$ is the vector of a trivial translation by the periods of Bravais lattice.

The matrices $D_{\mathbf{k}}(h)$ and their characters $\chi_{D_{\mathbf{k}}(h)}$ are determined from the formulas

$D_{\mathbf{k}}(h)=e^{-i k(\boldsymbol{\alpha}+\mathbf{a})} w(r) D(r)$

and

$\chi_{D_{\mathbf{k}}(h)}=e^{-i k(\boldsymbol{\alpha}+\mathbf{a})} w(r) \chi_{D(r)}$,

respectively. Here, in the case of representations that describe states neglecting their spin (with an integer spin), $w(r)=u(r) \equiv u_{1, \mathbf{k}}(r)$ is a function that transforms the factor-system $\omega\left(r_{2}, r_{1}\right) \equiv$ $\equiv \omega_{1, \mathbf{k}}\left(r_{2}, r_{1}\right)$, which is determined by the properties of the spatial group of the crystal, to the standard form. In the case of representations describing the states making allowance for their spin (with a halfinteger spin), $w(r)=u_{s}(r) \equiv u_{1, \mathbf{k}}(r) u_{2}(r)$ is a function that transforms the factor-system $\omega\left(r_{2}, r_{1}\right)=$ $=\omega_{s}\left(r_{2}, r_{1}\right)=\omega_{1, \mathbf{k}}\left(r_{2}, r_{1}\right) \omega_{2}\left(r_{2}, r_{1}\right)$, which is determined by the spinor transformations in the spatial group, and $u_{2}(r)$ is a function that transforms the factor-system $\omega_{2}\left(r_{2}, r_{1}\right)$, which is determined by the spinor transformations at the symmetry group operations with the group of wave-vector directions $F_{\mathbf{k}}$, to the standard form $\omega_{2}^{\prime}\left(r_{2}, r_{1}\right)$. In addition, $D(r)$ are irreducible representations corresponding to the standard factor-systems of the projective class, to which the factor-system $\omega\left(r_{2}, r_{1}\right)$ belongs, and $\chi_{D(r)}$ are the characters of irreducible projective representations $D(r)$.

The factor-system $\omega_{1}\left(r_{2}, r_{1}\right) \equiv \omega_{1, \mathbf{k}}\left(r_{2}, r_{1}\right)$ is constructed according to the formula [6]

$\omega_{1, \mathbf{k}}\left(r_{2}, r_{1}\right)=\exp \left[i\left(\mathbf{k}-r_{2}^{-1} \mathbf{k}\right) \boldsymbol{\alpha}_{1}\right]$,

so that no difficulty arises at any point in the Brillouin zone. It is also easy to determine the projective class, to which this factor-system belongs [6].

The transformation of spinors, i.e. the wave functions of states with a half-integer spin, is characterized by double-valued representations. For their construction, the Bethe method is often applied [7]. It is based on the introduction of an additional, abstract from the geometric viewpoint, symmetry element $q$, which commutes with all symmetry operations and is interpreted as a rotation of the quantum-mechanical system by an angle of $2 \pi$ around an arbitrary axis 1. This element changes the sign of the spinor, which characterizes the quantum states, into the opposite one. That is why the corresponding representation of the symmetry group is called two-valued. The next step in the Bethe method consists in the construction of a double group containing $2 n$ elements: $n$ "rotational" symmetry elements $r$ and $n$ additional elements $q r$. Next, the irreducible representations of the double group are found. Those of them, which are additional to the representations of the ordinary group, are the sought two-valued representations of the ordinary symmetry group. This method of constructing two-valued representations does not take into account that the latter should be projective representations of the ordinary symmetry group, and there is no need to construct a double abstract Bethe group for their construction. We only need to determine the factorsystems $\omega_{2}\left(r_{2}, r_{1}\right)$, which reflect the transformations 
of the wave functions of quantum-mechanical systems with a half-integer spin.

The factor-system $\omega_{2}\left(r_{2}, r_{1}\right)$ is defined as follows:

$\omega_{2}\left(r_{2}, r_{1}\right)= \begin{cases}1 & \text { if } 0 \leqslant \vartheta<2 \pi, \\ -1 & \text { if } 2 \pi \leqslant \vartheta<4 \pi,\end{cases}$

where $\vartheta$ is the angle of the rotation corresponding to the element product $r_{2} r_{1}$. The projective class, to which the factor-system belongs, can also be easily determined [6].

Let us define a standard factor-system for the projective representations of the projective class $K_{i}$.

As the standard factor-systems for projective representations of various projective classes $K_{i}$ of noncommutative (non-Abelian) point symmetry groups of the order $h$, we will call the factor-systems $\omega^{\prime}\left(r_{2}, r_{1}\right)$ consisting of real coefficients equal to 1 or -1 and containing the maximum number of periods in the elements $r_{2}$, which form adjacent classes forming the factor-groups according to the corresponding invariant subgroups.

Let us take into account the following circumstances. First, the standard factor-systems for the representations of the projective class $K_{0}$ completely consist of the coefficients equal to 1 . Secondly, the matrices of the irreducible representations corresponding to standard factor-systems are known for both the class $K_{0}$ (in this case, they coincide with ordinary vector representations) and the class $K_{1}$ (in this case, they can be easily calculated according to the transformations of the angular momentum with a half-integer quantum number $j$ ). Therefore, the construction of irreducible projective representations of the class $K_{1}$ of complete spatial symmetry groups - in particular, spinor representations is reduced to the construction of the factor-systems $\omega_{2}\left(r_{2}, r_{1}\right)$, the determination of the forms for the standard factor-systems of the class $K_{1}$, and the determination of the functions $u_{2}(r)$ that transform the factor-systems $\omega_{2}\left(r_{2}, r_{1}\right)$ to the $p$-equivalent standard form $\omega_{2}^{\prime}\left(r_{2}, r_{1}\right)$.

\section{Construction of Factor-Systems} $\omega_{2}\left(r_{2}, r_{1}\right)$ that Describe the Transformation of Spinors under the Action of Symmetry Operations, and their Reduction to the Standard Form $\omega_{2}^{\prime}\left(r_{2}, r_{1}\right)$

Let us construct a factor-system $\omega_{2}\left(r_{2}, r_{1}\right)$ that describes the transformation of spin variables for the symmetry group $6 / \mathrm{mmm}\left(D_{6 h}\right)$. As the $6 / \mathrm{mmm}$ group generators, we select the elements

$a=c_{3}, \quad b=\left(u_{2}\right)_{1}, \quad c=c_{2}, \quad d=i$.

This choice of generators makes allowance for the composition principle. According to the latter, the group $6 / \mathrm{mmm}$ can be represented as the direct product of the groups 622 and $\overline{1}$ (i.e. $6 / \mathrm{mmm}=622 \times \overline{1}$ ); then, the group 622 as the direct product of the groups 32 and 2 (i.e. $622=32 \times 2$ ), and the group 32 as the direct product of groups 3 and $2^{\prime}$ (i.e. $32=3 \times 2^{\prime}$ ).

By applying the generating relations, which are satisfied by the selected generating elements, let us calculate all values for $\omega_{2}\left(r_{2}, r_{1}\right)$. It is important that, for this operation, we should use the generating relations for the double group $(6 / \mathrm{mmm})^{\prime}$ :

$a^{6}=e, \quad b^{4}=e, \quad c^{4}=e, \quad d^{2}=e$,

$a b=q b a^{2}, \quad a c=c a, \quad a d=d a$,

$b c=q c b, \quad b d=d b, \quad c d=d c$.

While finding algebraic expressions for the elements of the double group $(6 / \mathrm{mmm})^{\prime}$ in terms of its generating elements, let us apply the following relation, which is general for the elements of the infinite double group of rotations $K^{\prime}[6,8]$ :

$f^{-1} c_{\mathbf{l}}(\alpha) f=c_{f^{-1} \mathbf{l}}(\alpha)$.

Here, $c_{\mathbf{l}}(\alpha)$ means a rotation by the angle $\alpha$ around the axis $\mathbf{l}$, and $f$ is an arbitrary rotation in the double rotation group, with the unity operation being considered as a rotation by an angle of $4 \pi$. It is relation (12) that allows the generating permutation relations indicated above and the algebraic expressions for the elements of the double group in terms of its generating elements [8] to be obtained. For example, for the double-group element $\left(u_{2}\right)_{2}$, we obtain $c_{3}\left(u_{2}\right)_{1} q c_{3}^{2}=\left(u_{2}\right)_{2}$, which brings us to the expression

$\left(u_{2}\right)_{2}=q a b a^{2}=q q b a^{2} a^{2}=b a^{4}=q b a$.

Note that another choice of generating elements can be used, but then the generating relations for them will also be changed. One can easily see that, provided the generating elements (11), the following algebraic expressions are obtained for the elements of the double group $(6 / m m m)^{\prime}: e=d^{0} c^{0} b^{0} a^{0}$,

ISSN 2071-0186. Ukr. J. Phys. 2018. Vol. 63, No. 5 
$c_{3}=d^{0} c^{0} b^{0} a^{1}, c_{3}^{2}=d^{0} c^{0} b^{0} a^{2}, \quad\left(u_{2}\right)_{1}=d^{0} c^{0} b^{1} a^{0}$, $\left(u_{2}\right)_{2}=q d^{0} c^{0} b^{1} a^{1},\left(u_{2}\right)_{3}=d^{0} c^{0} b^{1} a^{2}, c_{2}=d^{0} c^{1} b^{0} a^{0}$, $c_{6}^{5}=d^{0} c^{1} b^{0} a^{1}, c_{6}=q d^{0} c^{1} b^{0} a^{2},\left(u_{2}^{\prime}\right)_{1}=d^{0} c^{1} b^{1} a^{0}$, $\left(u_{2}^{\prime}\right)_{2}=q d^{0} c^{1} b^{1} a^{1},\left(u_{2}^{\prime}\right)_{3}=d^{0} c^{1} b^{1} a^{2}, i=d^{1} c^{0} b^{0} a^{0}$, $i c_{3}=d^{1} c^{0} b^{0} a^{1}, i c_{3}^{2}=d^{1} c^{0} b^{0} a^{2}, i\left(u_{2}\right)_{1}=\left(\sigma_{d}\right)_{1}=$ $=d^{1} c^{0} b^{1} a^{0}, i\left(u_{2}\right)_{2}=\left(\sigma_{d}\right)_{2}=q d^{1} c^{0} b^{1} a^{1}, i\left(u_{2}\right)_{3}=$ $=\left(\sigma_{d}\right)_{3}=d^{1} c^{0} b^{1} a^{2}, \quad i c_{2}=\sigma_{h}=d^{1} c^{1} b^{0} a^{0}, \quad i c_{6}^{5}=$ $=d^{1} c^{1} b^{0} a^{1}, i c_{6}=q d^{1} c^{1} b^{0} a^{2}, i\left(u_{2}^{\prime}\right)_{1}=\left(\sigma_{d}^{\prime}\right)_{1}=$ $=d^{1} c^{1} b^{1} a^{0}, i\left(u_{2}^{\prime}\right)_{2}=\left(\sigma_{d}^{\prime}\right)_{2}=q d^{1} c^{1} b^{1} a^{1}, i\left(u_{2}^{\prime}\right)_{3}=$ $=\left(\sigma_{d}^{\prime}\right)_{3}=d^{1} c^{1} b^{1} a^{2}$. The factor-system $\omega_{2}\left(r_{2}, r_{1}\right)$ calculated in such a way for the group $6 / \mathrm{mmm}$ is presented in Table 1. It belongs to the projective class $K_{1}$, because $\alpha=-1, \beta=1$, and $\gamma=1$ for it. The subscripts in parentheses near the coefficient values for the factor-system $\omega_{2}\left(r_{2}, r_{1}\right)$ correspond to the multiplication table for the elements of the group $6 / \mathrm{mmm}$ (numbers in the parentheses indicate the numerical notation of the elements corresponding to the products $r_{2} r_{1}$ ).

It should be noted that O.V. Kovalev $[1,2]$ made an attempt to construct this factor-system in the form of a multiplication table for the spinors in the group $6 / \mathrm{mmm}$. However, his attempt can be considered as a failure, because 216 of 576 values tabulated in works $[1,2]$ have the opposite sign. The matter is that the expression for the spinor wave function of a quantum-mechanical system with a half-integer spin contains an additional factor $c$ [9]. This factor can accept either of two values: +1 or -1 . Really, when being rotated by an angle of $2 \pi$ around any axis, the spinor changes its sign to the opposite one. This is the essence of the two-valued character of the representation matrix for every "rotational" symmetry element. Without restricting the general character of consideration, such a behavior is allowed only for the "rotational" generating symmetry elements of the crystalline class group. For other symmetry elements that are products of various powers of group generators, this factor cannot anymore accept any of two values. It always accepts the value following from the relation that defines this element. In other words, the arbitrary choice of the "internal" spinor sign for group generators does not affect the values of coefficients of the factor-system. Unfortunately, in works [1,2], the sign of a spinor at a transformation with the help of any "rotational" group element of the crystalline class was determined by algebraic expressions for the geometric image of the element in the ordinary, onevalued at rotations, three-dimensional space; i.e. for every of the nongenerate "rotational" group elements, it was determined arbitrarily.

The calculation of the factor-system $\omega_{2}\left(r_{2}, r_{1}\right)$ within our method is correct because, firstly, using transformation (5) and the coefficients $u_{2}(r)$ indicated in the lower part of Table 1 and calculated by formulas (13.3), (14.18), and (14.19) in work [6], it is reduced to the $p$-equivalent block-symmetric form, which corresponds to the definition of the standard factor-system, i.e. the factor-system $\omega_{2}^{\prime}\left(r_{2}, r_{1}\right)$. Second, when transforming the matrices of irreducible projective representations and their characters corresponding to the standard factor-system (they can be obtained independently by the method presented in work [6] for the extended group construction, i.e. a representation group, where they are determined by its unique irreducible representations and are complementary to the ordinary irreducible vector representations [6]) according to formula (4), the same coefficients $u_{2}(r)$ bring about the matrices and the characters of spinor representations that are identical to those obtained by means of the double group with the use of the Bethe method. Therefore, the factorsystem $\omega_{2}^{\prime}\left(r_{2}, r_{1}\right)$ is a true standard factor-system of the projective class $K_{1}$ of the group $6 / \mathrm{mmm}$.

Knowing the form of the standard factor-system $\omega_{2}^{\prime}\left(r_{2}, r_{1}\right)$ for the class $K_{1}$, we can construct standard factor-systems and, hence, irreducible projective representations for all projective classes. It will be done below.

Since the factor-systems $\omega_{2}\left(r_{2}, r_{1}\right)$ and $\omega_{2}^{\prime}\left(r_{2}, r_{1}\right)$ for the symmetry group $6 / \mathrm{mmm}$ belong to the projective class $K_{1}$, the standard factor-system $\omega_{2}^{\prime}\left(r_{2}, r_{1}\right)$ for this group coincides with the standard factorsystem $\omega_{(1)}^{\prime}\left(r_{2}, r_{1}\right)\left[\omega_{2}^{\prime}\left(r_{2}, r_{1}\right) \equiv \omega_{(1)}^{\prime}\left(r_{2}, r_{1}\right)\right]$, where the parenthesized subscript of the standard factorsystem means its projective class. The standard expression for the factor-system $\omega_{2}\left(r_{2}, r_{1}\right)$, which is denoted as $\omega_{2}^{\prime}\left(r_{2}, r_{1}\right)$ [for the group $6 / \mathrm{mmm}$, we have $\left.\omega_{2}^{\prime}\left(r_{2}, r_{1}\right)=\omega_{(1)}^{\prime}\left(r_{2}, r_{1}\right)\right]$, is shown in Table 2 . In Table 2 , an additional partitioning of the symmetry elements of the group $6 / \mathrm{mmm}$ is used: horizontally into blocks $a, b, c$, and $d$; and vertically into blocks $a\left(a_{1}, a_{2}\right), b\left(b_{1}, b_{2}\right), c\left(c_{1}, c_{2}\right)$, and $d\left(d_{1}, d_{2}\right)$. This partitioning allows a compact form (it is applied below) to be used to write down factor-systems, in which the coefficients with the same value in every block are substituted by the value of one coefficient, which is the same for all coefficients in the block. In Ta- 


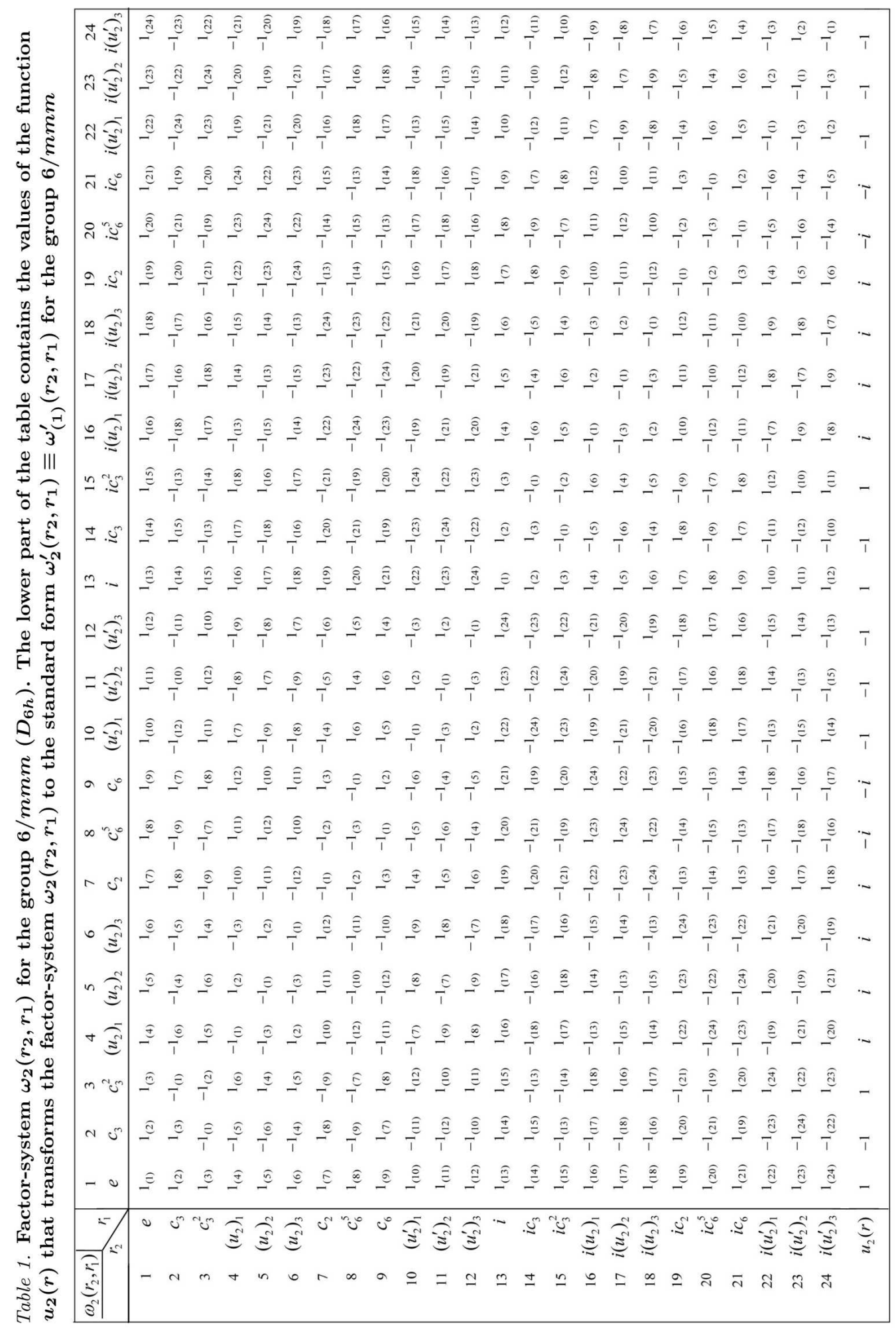


ble 2 and below, continuous lines are used to mark the contours of such blocks with the coefficients having a value of -1 .

\section{Characters of Irreducible}

Representations of the Double Group

$(6 / \mathrm{mmm})^{\prime}$ and the Irreducible Projective

Representations of the Group $6 / \mathrm{mmm}$, Which Correspond to Two-Valued Projective Representations of the Class $K_{1}$, Spinor

Ones and Those Corresponding to the Standard Factor-System

The characters of irreducible representations of the double group $(6 / \mathrm{mmm})^{\prime}\left(D_{6 h}^{\prime}\right)$ are quoted in Table 3 . It is their complementary single-valued irreducible representations (complementary to ordinary vector single-valued nonnegative representations of the group $6 / \mathrm{mmm}$, which can be obtained from the representations of the group $(6 / \mathrm{mmm})^{\prime}$ by a simple cancellation of the element $q$ from all relations) that are two-valued projective or spinor representations of the group $6 / \mathrm{mmm}$. The spinor representations are marked by the symbols $E_{1}^{\prime}, E_{2}^{\prime}$, and $E_{3}^{\prime}$ in the Mulliken notation or by the symbols $\Gamma_{7}, \Gamma_{8}$, and $\Gamma_{9}$ in the Koster notation, where the letter $\Gamma$ denotes not only their membership to a certain point group (in the given case, this is the group $6 / \mathrm{mmm}$ ), but also to the coinciding group of directions of the wavevector group of point $\Gamma$ in crystals or periodic nanostructures. In the form of projective representations, these irreducible representations and their characters can also be obtained by formulas (6) and (7), where $D(r)$ and $\chi_{\left.D_{(} r\right)}$ are projective representations and their characters, respectively, which correspond to the standard factor-systems of those classes that include the factor-systems $\omega\left(r_{2}, r_{1}\right)$.

The characters of irreducible representations of the projective classes $K_{0}$ (ordinary single-valued or vector ones) and $K_{1}$ (two-valued projective or spinor ones) are quoted in Table 4 . It is easy to see that the characters of irreducible projective representations of the class $K_{1}$ of the group $6 / \mathrm{mmm}$ coincide with the characters of spinor irreducible representations of the double group $(6 / \mathrm{mmm})^{\prime}$.

The characters of the projective representations of the projective class $K_{1}$ that correspond to the standard factor-system $\omega_{(1)}^{\prime}\left(r_{2}, r_{1}\right)$ can be obtained, as was mentioned above, by the method of constructing a group of representations for the group $6 / \mathrm{mmm}$
[6] or, which is much simpler, using formula (4) with the substituted values for the coefficients $u_{2}(r)$, which bring the factor-system $\omega_{2}\left(r_{2}, r_{1}\right)$ to the standard form $\omega_{(1)}^{\prime}\left(r_{2}, r_{1}\right)$. The characters of the irreducible projective representations of the projective classes $K_{0}$ and $K_{1}$ of the group $6 / \mathrm{mmm}$ that correspond to the standard factor-systems $\omega_{(0)}^{\prime}\left(r_{2}, r_{1}\right)$ and $\omega_{(1)}^{\prime}\left(r_{2}, r_{1}\right)$, respectively, are quoted in Table 5 , with the characters of two-dimensional projective representations that correspond to the standard factor-system $\omega_{(1)}^{\prime}\left(r_{2}, r_{1}\right)$, being designated by the symbol $P$.

\section{Factor-Systems Associated} with the Structure of Spatial Symmetry Groups. Their Construction and Reduction to the Standard Form. Determination of Projective Classes for Vibrational and Electron Excitations at Various Points in the Brillouin Zone

Now, let us use formula (8) to construct the factorsystem $\omega_{1, A}\left(r_{2}, r_{1}\right)$. The latter is determined by the properties of the spatial symmetry group of a graphite crystal and is responsible for single-valued irreducible projective representations of point $A$. The corresponding result is presented in Table 6 . After calculating the values of the coefficients $\alpha, \beta$, and $\gamma$, one can easily see that the constructed factor-system belongs to the projective class $K_{5}$. The values of the function on the group $u_{1, A}(r)$, which bring the factorsystem $\omega_{1, A}\left(r_{2}, r_{1}\right)$ to the standard form $\omega_{1, A}^{\prime}\left(r_{2}, r_{1}\right)$, can be easily found by the method described in work [6, formulas (14.33)] and in works [10-12]. They are exhibited in the lower part of Table 6 .

Table 7 illustrates the standard factor-system of the projective class $K_{5}$, i.e. the factor-system $\omega_{1, A}^{\prime}\left(r_{2}, r_{1}\right) \equiv \omega_{(5)}^{\prime}\left(r_{2}, r_{1}\right)$, which can be easily obtained making use of the values of the function $u_{1, A}(r)$ and formula (5). The characters of one-valued irreducible projective representations for point $A$ are also easy to be obtained, if we know the values of the function $u_{1, A}(r)$ and the characters of irreducible projective representations of the class $K_{5}$ corresponding to the standard factor-system of the class $K_{5}$, which are given in Table 8 [6]. The single-valued irreducible projective representations for point $A$ are given in Table 9 (the first three irreducible projective representations).

It is easy to get convinced that the two-valued (spinor) irreducible projective representations at 


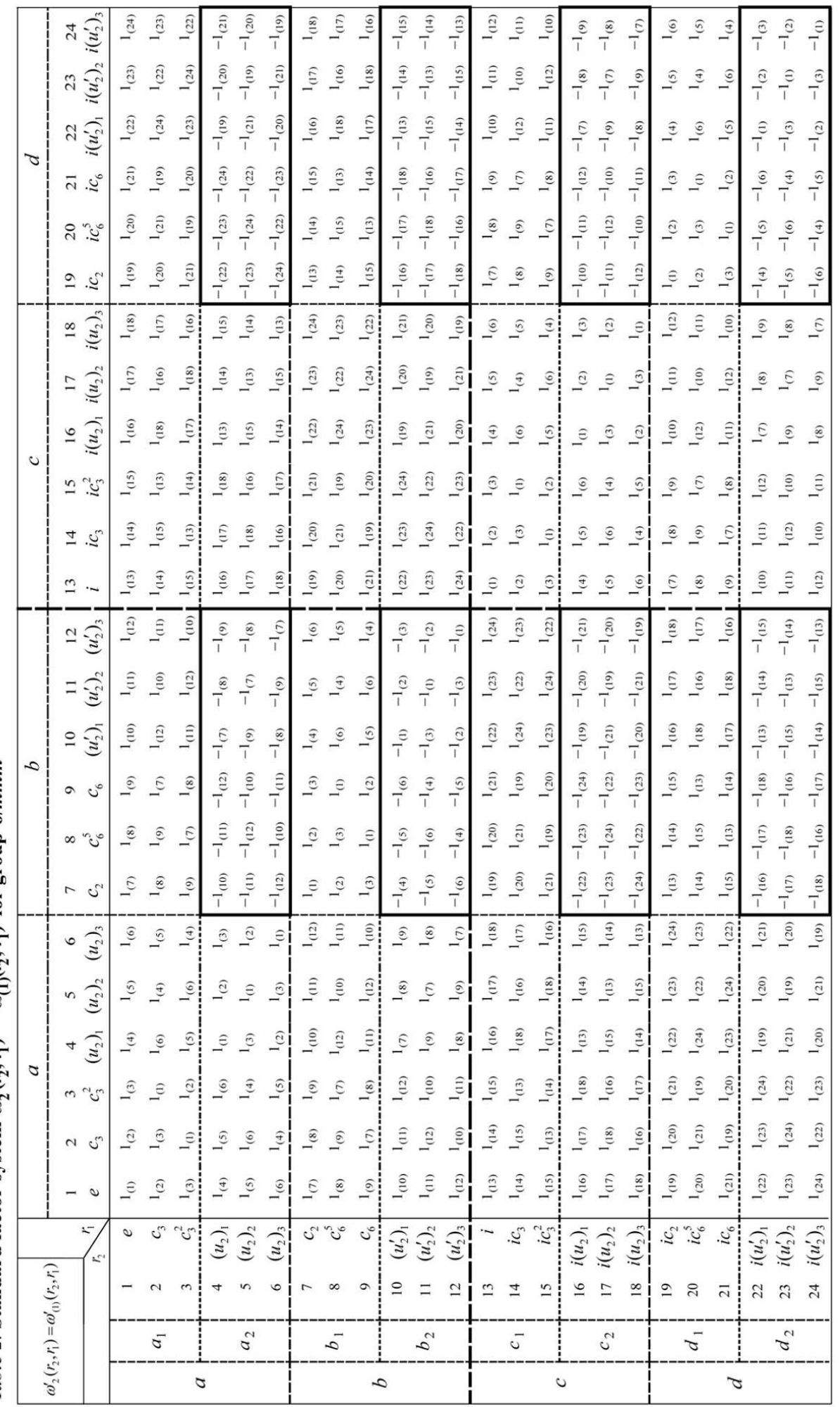


Table 3. Characters of irreducible representations of the double group $(6 / \mathrm{mmm})^{\prime}\left(D_{6 h}^{\prime}\right)$

\begin{tabular}{|c|c|c|c|c|c|c|c|c|c|c|c|c|c|c|c|c|c|c|c|}
\hline$(6 / m m m)^{\prime}$ & $\left(D_{6 h}^{\prime}\right)$ & $e$ & $q$ & $\begin{array}{l}c_{3}, \\
q c_{3}^{2}\end{array}$ & $\begin{array}{l}c_{3}^{2}, \\
q c_{3}\end{array}$ & $\begin{array}{l}3 u_{2}, \\
3 q u_{2}\end{array}$ & $\begin{array}{l}c_{2}, \\
q c_{2}\end{array}$ & $\begin{array}{l}c_{6}^{5} \\
q c_{6}\end{array}$ & $\begin{array}{l}c_{6}, \\
q c_{6}^{5}\end{array}$ & $\begin{array}{c}3 u_{2}^{\prime} \\
3 q u_{2}^{\prime}\end{array}$ & $i$ & $q i$ & $\begin{array}{l}i c_{3}, \\
q i c_{3}^{2}\end{array}$ & $\begin{array}{l}i c_{3}^{2} \\
q i c_{3}\end{array}$ & $\begin{array}{l}3 \sigma_{d} \\
3 q \sigma_{d}\end{array}$ & $\begin{array}{l}\sigma_{h} \\
q \sigma_{h}\end{array}$ & $\begin{array}{l}i c_{6}^{5} \\
q i c_{6}\end{array}$ & $\begin{array}{l}i c_{6}, \\
q i c_{6}^{5}\end{array}$ & $\begin{array}{l}3 \sigma_{d}^{\prime} \\
3 q \sigma_{d}^{\prime}\end{array}$ \\
\hline$\Gamma_{1}^{+}$ & $A_{1}^{+}$ & 1 & 1 & 1 & 1 & 1 & 1 & 1 & 1 & 1 & 1 & 1 & 1 & 1 & 1 & 1 & 1 & 1 & 1 \\
\hline$\Gamma_{1}^{-}$ & $A_{1}^{-}$ & 1 & 1 & 1 & 1 & 1 & 1 & 1 & 1 & 1 & -1 & -1 & -1 & -1 & -1 & -1 & -1 & -1 & -1 \\
\hline$\Gamma_{2}^{+}$ & $A_{2}^{+}$ & 1 & 1 & 1 & 1 & 1 & -1 & -1 & -1 & -1 & 1 & 1 & 1 & 1 & 1 & -1 & -1 & -1 & -1 \\
\hline$\Gamma_{2}^{-}$ & $A_{2}^{-}$ & 1 & 1 & 1 & 1 & 1 & -1 & -1 & -1 & -1 & -1 & -1 & -1 & -1 & -1 & 1 & 1 & 1 & 1 \\
\hline$\Gamma_{3}^{+}$ & $A_{3}^{+}$ & 1 & 1 & 1 & 1 & -1 & 1 & 1 & 1 & -1 & 1 & 1 & 1 & 1 & -1 & 1 & 1 & 1 & -1 \\
\hline$\Gamma_{3}^{-}$ & $A_{3}^{-}$ & 1 & 1 & 1 & 1 & -1 & 1 & 1 & 1 & -1 & -1 & -1 & -1 & -1 & 1 & -1 & -1 & -1 & 1 \\
\hline$\Gamma_{4}^{+}$ & $A_{4}^{+}$ & 1 & 1 & 1 & 1 & -1 & -1 & -1 & -1 & 1 & 1 & 1 & 1 & 1 & -1 & -1 & -1 & -1 & 1 \\
\hline$\Gamma_{4}^{-}$ & $A_{4}^{-}$ & 1 & 1 & 1 & 1 & -1 & -1 & -1 & -1 & 1 & -1 & -1 & -1 & -1 & 1 & 1 & 1 & 1 & -1 \\
\hline$\Gamma_{5}^{+}$ & $E_{1}^{+}$ & 2 & 2 & -1 & -1 & 0 & 2 & -1 & -1 & 0 & 2 & 2 & -1 & -1 & 0 & 2 & -1 & -1 & 0 \\
\hline$\Gamma_{5}^{-}$ & $E_{1}^{-}$ & 2 & 2 & -1 & -1 & 0 & 2 & -1 & -1 & 0 & -2 & -2 & 1 & 1 & 0 & -2 & 1 & 1 & 0 \\
\hline$\Gamma_{6}^{+}$ & $E_{2}^{+}$ & 2 & 2 & -1 & -1 & 0 & -2 & 1 & 1 & 0 & 2 & 2 & -1 & -1 & 0 & -2 & 1 & 1 & 0 \\
\hline$\Gamma_{6}^{-}$ & $E_{2}^{-}$ & 2 & 2 & -1 & -1 & 0 & -2 & 1 & 1 & 0 & -2 & -2 & 1 & 1 & 0 & 2 & -1 & -1 & 0 \\
\hline$\Gamma_{7}^{+}$ & $\left(E_{1}^{\prime}\right)^{+}$ & 2 & -2 & 1 & -1 & 0 & 0 & $\sqrt{3}$ & $-\sqrt{3}$ & 0 & 2 & -2 & 1 & -1 & 0 & 0 & $\sqrt{3}$ & $-\sqrt{3}$ & 0 \\
\hline$\Gamma_{7}^{-}$ & $\left(E_{1}^{\prime}\right)^{-}$ & 2 & -2 & 1 & -1 & 0 & 0 & $\sqrt{3}$ & $-\sqrt{3}$ & 0 & -2 & 2 & -1 & 1 & 0 & 0 & $-\sqrt{3}$ & $\sqrt{3}$ & 0 \\
\hline$\Gamma_{8}^{+}$ & $\left(E_{2}^{\prime}\right)^{+}$ & 2 & -2 & 1 & -1 & 0 & 0 & $-\sqrt{3}$ & $\sqrt{3}$ & 0 & 2 & -2 & 1 & -1 & 0 & 0 & $-\sqrt{3}$ & $\sqrt{3}$ & 0 \\
\hline$\Gamma_{8}^{-}$ & $\left(E_{2}^{\prime}\right)^{-}$ & 2 & -2 & 1 & -1 & 0 & 0 & $-\sqrt{3}$ & $\sqrt{3}$ & 0 & -2 & 2 & -1 & 1 & 0 & 0 & $\sqrt{3}$ & $-\sqrt{3}$ & 0 \\
\hline$\Gamma_{9}^{+}$ & $\left(E_{3}^{\prime}\right)^{+}$ & 2 & -2 & -2 & 2 & 0 & 0 & 0 & 0 & 0 & 2 & -2 & -2 & 2 & 0 & 0 & 0 & 0 & 0 \\
\hline$\Gamma_{9}^{-}$ & $\left(E_{3}^{\prime}\right)^{-}$ & 2 & -2 & -2 & 2 & 0 & 0 & 0 & 0 & 0 & -2 & 2 & 2 & -2 & 0 & 0 & 0 & 0 & 0 \\
\hline
\end{tabular}

Table 4. Characters of single- and two-valued irreducible projective representations of point $\boldsymbol{\Gamma}$

\begin{tabular}{|c|c|c|c|c|c|c|c|c|c|c|c|c|c|c|c|c|c|c|}
\hline \multirow{2}{*}{$\begin{array}{c}\text { Projec- } \\
\text { tive } \\
\text { class }\end{array}$} & \multirow{2}{*}{\multicolumn{2}{|c|}{$\begin{array}{c}\text { Indication } \\
\text { of an irreducible } \\
\text { projective } \\
\text { representation }\end{array}$}} & \multicolumn{16}{|c|}{$6 / m m m\left(D_{6 h}\right)$} \\
\hline & & & $e$ & $c_{3}$ & $c_{3}^{2}$ & $3 u_{2}$ & $c_{2}$ & $c_{6}^{5}$ & $c_{6}$ & $3 u_{2}^{\prime}$ & $i$ & $i c_{3}$ & $i c_{3}^{2}$ & $3 \sigma_{d}$ & $\sigma_{h}$ & $i c_{6}^{5}$ & $i c_{6}$ & $3 \sigma_{d}^{\prime}$ \\
\hline \multirow[t]{12}{*}{$K_{0}$} & $\Gamma_{1}^{+}$ & $A_{1}^{+}$ & 1 & 1 & 1 & 1 & 1 & 1 & 1 & 1 & 1 & 1 & 1 & 1 & 1 & 1 & 1 & 1 \\
\hline & & $A_{1}^{-}$ & 1 & 1 & 1 & 1 & 1 & 1 & 1 & 1 & -1 & -1 & -1 & -1 & -1 & -1 & -1 & -1 \\
\hline & & $A_{2}^{+}$ & 1 & 1 & 1 & 1 & -1 & -1 & -1 & -1 & 1 & 1 & 1 & 1 & -1 & -1 & -1 & -1 \\
\hline & & $A_{2}^{-}$ & 1 & 1 & 1 & 1 & -1 & -1 & -1 & -1 & -1 & -1 & -1 & -1 & 1 & 1 & 1 & 1 \\
\hline & $\Gamma_{3}^{+}$ & $A_{3}^{+}$ & 1 & 1 & 1 & -1 & 1 & 1 & 1 & -1 & 1 & 1 & 1 & -1 & 1 & 1 & 1 & -1 \\
\hline & & $A_{3}^{-}$ & 1 & 1 & 1 & -1 & 1 & 1 & 1 & -1 & -1 & -1 & -1 & 1 & -1 & -1 & -1 & 1 \\
\hline & $\Gamma_{4}^{+}$ & $A_{4}^{+}$ & 1 & 1 & 1 & -1 & -1 & -1 & -1 & 1 & 1 & 1 & 1 & -1 & -1 & -1 & -1 & 1 \\
\hline & & $A_{4}^{-}$ & 1 & 1 & 1 & -1 & -1 & -1 & -1 & 1 & -1 & -1 & -1 & 1 & 1 & 1 & 1 & -1 \\
\hline & & $E_{1}^{+}$ & 2 & -1 & -1 & 0 & 2 & -1 & -1 & 0 & 2 & -1 & -1 & 0 & 2 & -1 & -1 & 0 \\
\hline & & $E_{1}^{-}$ & 2 & -1 & -1 & 0 & 2 & -1 & -1 & 0 & -2 & 1 & 1 & 0 & -2 & 1 & 1 & 0 \\
\hline & & $E_{2}^{+}$ & 2 & -1 & -1 & 0 & -2 & 1 & 1 & 0 & 2 & -1 & -1 & 0 & -2 & 1 & 1 & 0 \\
\hline & & $E_{2}^{-}$ & 2 & -1 & -1 & 0 & -2 & 1 & 1 & 0 & -2 & 1 & 1 & 0 & 2 & -1 & -1 & 0 \\
\hline \multirow[t]{6}{*}{$K_{1}$} & $\Gamma_{7}^{+}$ & $\left(E_{1}^{\prime}\right)$ & 2 & 1 & -1 & 0 & 0 & $\sqrt{3}$ & $-\sqrt{3}$ & 0 & 2 & 1 & -1 & 0 & 0 & $\sqrt{3}$ & $-\sqrt{3}$ & 0 \\
\hline & & $\left(E_{1}^{\prime}\right)$ & 2 & 1 & -1 & 0 & 0 & $\sqrt{3}$ & $-\sqrt{3}$ & 0 & -2 & -1 & 1 & 0 & 0 & $-\sqrt{3}$ & $\sqrt{3}$ & 0 \\
\hline & $\Gamma_{8}^{+}$ & $\left(E_{2}^{\prime}\right)$ & 2 & 1 & -1 & 0 & 0 & $-\sqrt{3}$ & $\sqrt{3}$ & 0 & 2 & 1 & -1 & 0 & 0 & $-\sqrt{3}$ & $\sqrt{3}$ & 0 \\
\hline & & $\left(E_{2}^{\prime}\right)$ & 2 & 1 & -1 & 0 & 0 & $-\sqrt{3}$ & $\sqrt{3}$ & 0 & -2 & -1 & 1 & 0 & 0 & $\sqrt{3}$ & $-\sqrt{3}$ & 0 \\
\hline & $\Gamma_{9}^{+}$ & $\left(E_{3}^{\prime}\right)$ & 2 & -2 & 2 & 0 & 0 & 0 & 0 & 0 & 2 & -2 & 2 & 0 & 0 & 0 & 0 & 0 \\
\hline & & $\left(E_{3}^{\prime}\right)$ & 2 & -2 & 2 & 0 & 0 & 0 & 0 & 0 & -2 & 2 & -2 & 0 & 0 & 0 & 0 & 0 \\
\hline
\end{tabular}


Table 5. Characters of irreducible projective representations of the projective classes

$K_{0}$ and $K_{1}$ of the group $6 / \mathrm{mmm}$ corresponding to the standard factor-systems $\omega_{(0)}^{\prime}\left(r_{2}, r_{1}\right)$ and $\omega_{(1)}^{\prime}\left(r_{2}, r_{1}\right)$

\begin{tabular}{|c|c|c|c|c|c|c|c|c|c|c|c|c|c|c|c|c|c|}
\hline \multirow{2}{*}{$\begin{array}{l}\text { Projec- } \\
\text { tive } \\
\text { class }\end{array}$} & \multirow{2}{*}{$\begin{array}{c}\text { Indication } \\
\text { of an irreducible } \\
\text { projective } \\
\text { representation }\end{array}$} & \multicolumn{16}{|c|}{$6 / m m m\left(D_{6 h}\right)$} \\
\hline & & $e$ & $c_{1}$ & $c_{3}^{2}$ & $3 u_{2}$ & $c_{2}$ & $c_{6}^{5}$ & $c_{6}$ & $3 u_{2}^{\prime}$ & $i$ & $i c_{3}$ & $i c_{3}^{2}$ & $3 \sigma_{d}$ & $\sigma_{h}$ & $i c_{6}^{5}$ & $i c_{6}$ & $3 \sigma_{d}^{\prime}$ \\
\hline \multirow[t]{12}{*}{$K_{0}$} & $\Gamma_{1}^{+} \quad A_{1}^{+}$ & 1 & 1 & 1 & 1 & 1 & 1 & 1 & 1 & 1 & 1 & 1 & 1 & 1 & 1 & 1 & 1 \\
\hline & $\Gamma_{1}^{-} \quad A_{1}^{-}$ & 1 & 1 & 1 & 1 & 1 & 1 & 1 & 1 & -1 & -1 & -1 & -1 & -1 & -1 & -1 & -1 \\
\hline & $\begin{array}{ll}\Gamma_{2}^{+} & A_{2}^{+}\end{array}$ & 1 & 1 & 1 & 1 & -1 & -1 & -1 & -1 & 1 & 1 & 1 & 1 & -1 & -1 & -1 & -1 \\
\hline & $\Gamma_{2}^{-} \quad A_{2}^{-}$ & 1 & 1 & 1 & 1 & -1 & -1 & -1 & -1 & -1 & -1 & -1 & -1 & 1 & 1 & 1 & 1 \\
\hline & $\begin{array}{ll}\Gamma_{3}^{+} & A_{3}^{+}\end{array}$ & 1 & 1 & 1 & -1 & 1 & 1 & 1 & -1 & 1 & 1 & 1 & -1 & 1 & 1 & 1 & -1 \\
\hline & $\Gamma_{3}^{-} \quad A_{3}^{-}$ & 1 & 1 & 1 & -1 & 1 & 1 & 1 & -1 & -1 & -1 & -1 & 1 & -1 & -1 & -1 & 1 \\
\hline & $\begin{array}{cc}\Gamma_{4}^{+} & A_{4}^{+}\end{array}$ & 1 & 1 & 1 & -1 & -1 & -1 & -1 & 1 & 1 & 1 & 1 & -1 & -1 & -1 & -1 & 1 \\
\hline & $\Gamma_{4}^{-} \quad A_{4}^{-}$ & 1 & 1 & 1 & -1 & -1 & -1 & -1 & 1 & -1 & -1 & -1 & 1 & 1 & 1 & 1 & -1 \\
\hline & $\Gamma_{5}^{+} \quad E_{1}^{+}$ & 2 & -1 & -1 & 0 & 2 & -1 & -1 & 0 & 2 & -1 & -1 & 0 & 2 & -1 & -1 & 0 \\
\hline & $\Gamma_{5}^{-} \quad E_{1}^{-}$ & 2 & -1 & -1 & 0 & 2 & -1 & -1 & 0 & -2 & 1 & 1 & 0 & -2 & 1 & 1 & 0 \\
\hline & $\Gamma_{6}^{+} \quad E_{2}^{+}$ & 2 & -1 & -1 & 0 & -2 & 1 & 1 & 0 & 2 & -1 & -1 & 0 & -2 & 1 & 1 & 0 \\
\hline & $\Gamma_{6}^{-} \quad E_{2}^{-}$ & 2 & -1 & -1 & 0 & -2 & 1 & 1 & 0 & -2 & 1 & 1 & 0 & 2 & -1 & -1 & 0 \\
\hline \multirow[t]{6}{*}{$K_{1}$} & $\left(P_{1}^{(1)}\right)^{+}$ & 2 & -1 & -1 & 0 & 0 & $\sqrt{3} i$ & $-\sqrt{3} i$ & 0 & 2 & -1 & -1 & 0 & 0 & $\sqrt{3} i$ & $-\sqrt{3} i$ & 0 \\
\hline & $\left(P_{1}^{(1)}\right)^{-}$ & 2 & -1 & -1 & 0 & 0 & $\sqrt{3} i$ & $-\sqrt{3} i$ & 0 & -2 & 1 & 1 & 0 & 0 & $-\sqrt{3} i$ & $\sqrt{3} i$ & 0 \\
\hline & $\left(P_{2}^{(1)}\right)^{+}$ & 2 & -1 & -1 & 0 & 0 & $-\sqrt{3} i$ & $\sqrt{3} i$ & 0 & 2 & -1 & -1 & 0 & 0 & $-\sqrt{3} i$ & $\sqrt{3} i$ & 0 \\
\hline & $\left(P_{2}^{(1)}\right)^{-}$ & 2 & -1 & -1 & 0 & 0 & $-\sqrt{3} i$ & $\sqrt{3} i$ & 0 & -2 & 1 & 1 & 0 & 0 & $\sqrt{3} i$ & $-\sqrt{3} i$ & 0 \\
\hline & $\left(P_{3}^{(1)}\right)^{+}$ & 2 & 2 & 2 & 0 & 0 & 0 & 0 & 0 & 2 & 2 & 2 & 0 & 0 & 0 & 0 & 0 \\
\hline & $\left(P_{3}^{(1)}\right)^{-}$ & 2 & 2 & 2 & 0 & 0 & 0 & 0 & 0 & -2 & -2 & -2 & 0 & 0 & 0 & 0 & 0 \\
\hline
\end{tabular}

point $A$ belong to the projective class $K_{4}$. Really, by multiplying the corresponding values of the constants $\alpha, \beta$, and $\gamma$, we obtain that, in our case, $K_{1} \times K_{5}=K_{1} \times K_{5}=K_{1} K_{5}=K_{4}$. The standard factor-system of the class $K_{4}$ is the factor-system $\omega_{(4)}^{\prime}\left(r_{2}, r_{1}\right)^{1}$ with the coefficients $\omega_{(4)}^{\prime}\left(r_{2}, r_{1}\right)=$ $=\omega_{(1)}^{\prime}\left(r_{2}, r_{1}\right) \omega_{(5)}^{\prime}\left(r_{2}, r_{1}\right)$. The corresponding characters of the two-valued (spinor) projective representations at point $A$, which are related to the projective class $K_{4}$, are given in Table 9 (the remaining three irreducible projective representations). The characters of those irreducible projective representations can be obtained by multiplying the values of the products $u_{1, A}(r) u_{2}(r)$ and the values of the characters of irreducible projective representations of class $K_{4}$ that correspond to the standard factor-system of the class $K_{4}$ (they are presented in Table 8 [6]).

\footnotetext{
${ }^{1}$ It is easy to see that $\omega_{(4)}^{\prime}\left(r_{2}, r_{1}\right)=\omega_{2, A}^{\prime}\left(r_{2}, r_{1}\right)=$ $=\omega_{1, A}^{\prime}\left(r_{2}, r_{1}\right) \omega_{2}^{\prime}\left(r_{2}, r_{1}\right)=\omega_{(5)}^{\prime}\left(r_{2}, r_{1}\right) \omega_{(1)}^{\prime}\left(r_{2}, r_{1}\right)=$ $=\omega_{(1)}^{\prime}\left(r_{2}, r_{1}\right) \omega_{(5)}^{\prime}\left(r_{2}, r_{1}\right)$, where $\omega_{2, A}^{\prime}\left(r_{2}, r_{1}\right)$ is a standard factor-system for the corresponding two-valued projective representations at point $A$.
}

Now, it is easy to determine the form of standard factor-systems for other projective classes of the group $6 / \mathrm{mmm}$. The standard factor-systems for all eight projective classes of the group $6 / \mathrm{mmm}$ are quoted in Table 10.

In the absence of external magnetic fields, additional conditions are imposed on the wave functions of states and, as a result, the representations. Those conditions are associated with the invariance with respect to the time inversion. Provided that they are satisfied, an additional degeneration may arise for some states.

\section{Symmetry of Lattice Vibrations and Electron $\pi$-Bands, and Their Dispersion in Single-Layer Graphene $C_{L 1}$ and Crystalline Graphite $\gamma-C$}

The representations of fundamental vibrations in crystal lattices $\left(\Gamma_{\text {lat. vibr }}\right)$ and the electron $\pi$-bands at $k=0\left(\Gamma_{\pi}\right)$ for single-layer graphene $C_{L 1}$ and crystalline graphite $\gamma-C$ are determined by the same point group $6 / \mathrm{mmm}\left(D_{6 h}\right)$. In the case of singlelayer graphene, this group describes csymmetry of 


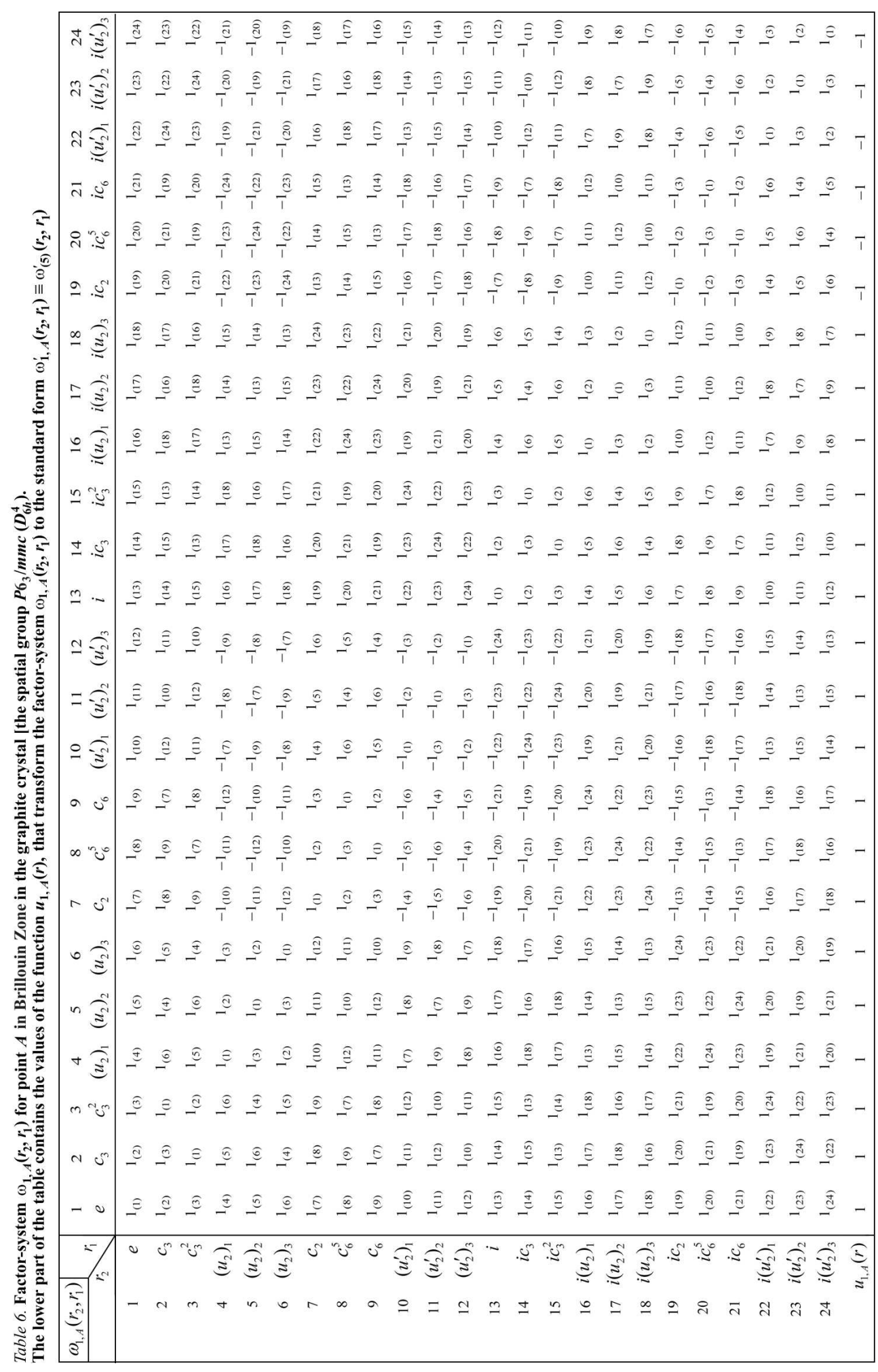




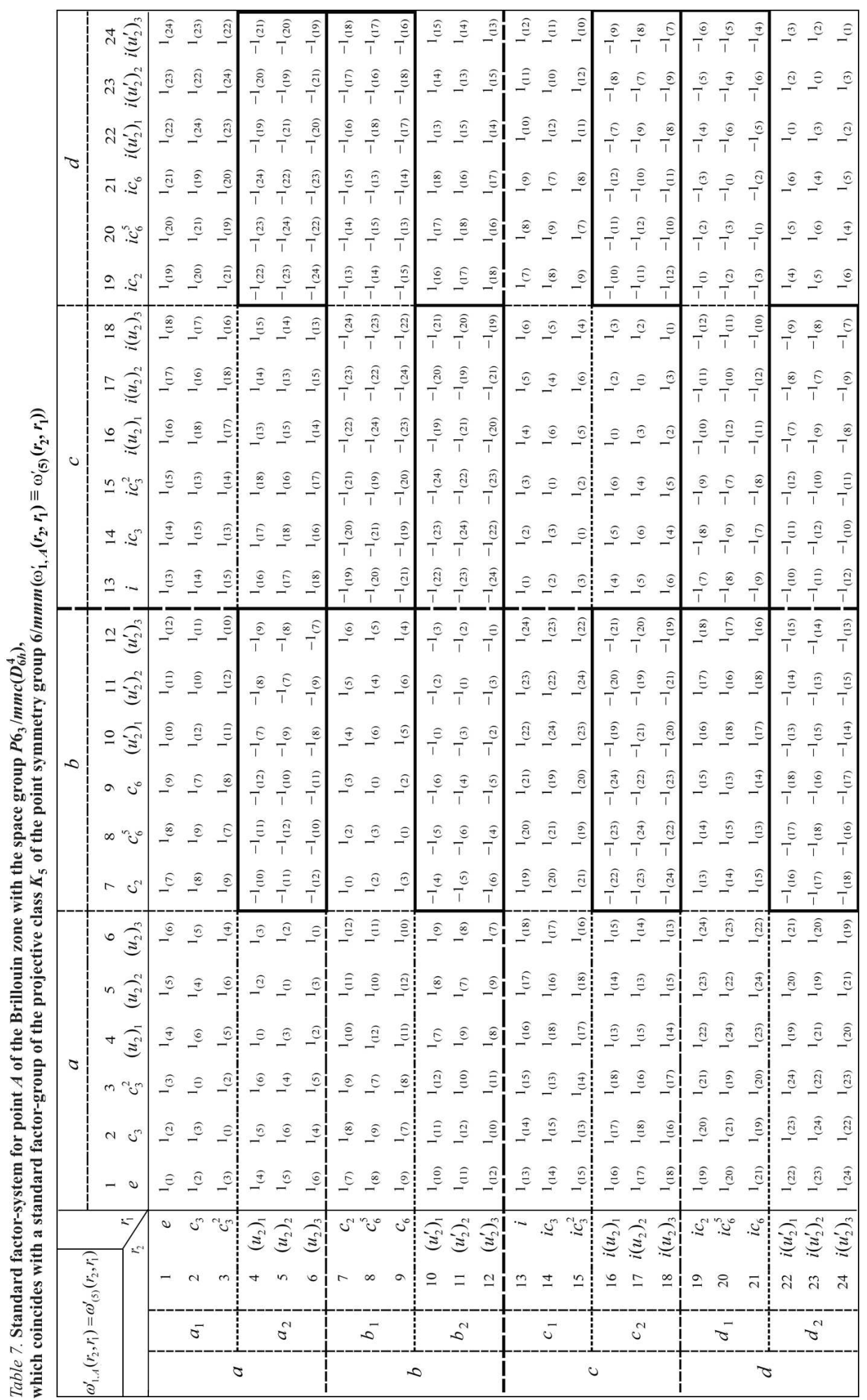


Table 8. Characters of irreducible projective representations of the projective classes $\boldsymbol{K}_{\mathbf{5}}$ and $\boldsymbol{K}_{4}$ of the group $6 / \mathrm{mmm}$ corresponding to the standard factor-systems of those classes $\omega_{(5)}^{\prime}\left(r_{2}, r_{1}\right)$ and $\omega_{(4)}^{\prime}\left(r_{2}, r_{1}\right)$

\begin{tabular}{|c|c|c|c|c|c|c|c|c|c|c|c|c|c|c|c|c|c|}
\hline \multirow{2}{*}{$\begin{array}{c}\text { Projec- } \\
\text { tive } \\
\text { class }\end{array}$} & \multirow{2}{*}{$\begin{array}{c}\text { Indication } \\
\text { of an irreducible } \\
\text { projective } \\
\text { representation }\end{array}$} & \multicolumn{16}{|c|}{$6 / m m m\left(D_{6 h}\right)$} \\
\hline & & $e$ & $c_{1}$ & $c_{3}^{2}$ & $3 u_{2}$ & $c_{2}$ & $c_{6}^{5}$ & $c_{6}$ & $3 u_{2}^{\prime}$ & $i$ & $i c_{3}$ & $i c_{3}^{2}$ & $3 \sigma_{d}$ & $\sigma_{h}$ & $i c_{6}^{5}$ & $i c_{6}$ & $3 \sigma_{d}^{\prime}$ \\
\hline \multirow[t]{3}{*}{$K_{5}$} & $P_{1}^{(5)}$ & 2 & 2 & 2 & 0 & 0 & 0 & 0 & 0 & 0 & 0 & 0 & 2 & 0 & 0 & 0 & 0 \\
\hline & $P_{2}^{(5)}$ & 2 & 2 & 2 & 0 & 0 & 0 & 0 & 0 & 0 & 0 & 0 & -2 & 0 & 0 & 0 & 0 \\
\hline & $Q^{(5)}$ & 4 & -2 & -2 & 0 & 0 & 0 & 0 & 0 & 0 & 0 & 0 & 0 & 0 & 0 & 0 & 0 \\
\hline \multirow[t]{3}{*}{$K_{4}$} & $P_{1}^{(4)}$ & 2 & 2 & 2 & 2 & 0 & 0 & 0 & 0 & 0 & 0 & 0 & 0 & 0 & 0 & 0 & 0 \\
\hline & $P_{2}^{(4)}$ & 2 & 2 & 2 & -2 & 0 & 0 & 0 & 0 & 0 & 0 & 0 & 0 & 0 & 0 & 0 & 0 \\
\hline & $Q^{(4)}$ & 4 & -2 & -2 & 0 & 0 & 0 & 0 & 0 & 0 & 0 & 0 & 0 & 0 & 0 & 0 & 0 \\
\hline
\end{tabular}

Table 9. Characters of single- and two-valued irreducible projective representations of point $A$

\begin{tabular}{|c|c|c|c|c|c|c|c|c|c|c|c|c|c|c|c|c|c|}
\hline \multirow{2}{*}{$\begin{array}{c}\text { Projec- } \\
\text { tive } \\
\text { class }\end{array}$} & \multirow{2}{*}{$\begin{array}{c}\text { Indication } \\
\text { of an irreducible } \\
\text { projective } \\
\text { representation }\end{array}$} & \multicolumn{16}{|c|}{$6 / m m m\left(D_{6 h}\right)$} \\
\hline & & $e$ & $c_{1}$ & $c_{3}^{2}$ & $3 u_{2}$ & $c_{2}$ & $c_{6}^{5}$ & $c_{6}$ & $3 u_{2}^{\prime}$ & $i$ & $i c_{3}$ & $i c_{3}^{2}$ & $3 \sigma_{d}$ & $\sigma_{h}$ & $i c_{6}^{5}$ & $i c_{6}$ & $3 \sigma_{d}^{\prime}$ \\
\hline \multirow[t]{3}{*}{$K_{5}$} & $A_{1}$ & 2 & 2 & 2 & 0 & 0 & 0 & 0 & 0 & 0 & 0 & 0 & 2 & 0 & 0 & 0 & 0 \\
\hline & $A_{2}$ & 2 & 2 & 2 & 0 & 0 & 0 & 0 & 0 & 0 & 0 & 0 & -2 & 0 & 0 & 0 & 0 \\
\hline & $A_{3}$ & 4 & -2 & -2 & 0 & 0 & 0 & 0 & 0 & 0 & 0 & 0 & 0 & 0 & 0 & 0 & 0 \\
\hline \multirow[t]{3}{*}{$K_{4}$} & $A_{4}, A_{4}$ & 2 & -2 & 2 & $2 i$ & 0 & 0 & 0 & 0 & 0 & 0 & 0 & 0 & 0 & 0 & 0 & 0 \\
\hline & $A_{5}$ & 2 & -2 & 2 & $-2 i$ & 0 & 0 & 0 & 0 & 0 & 0 & 0 & 0 & 0 & 0 & 0 & 0 \\
\hline & $A_{6}$ & 4 & 2 & -2 & 0 & 0 & 0 & 0 & 0 & 0 & 0 & 0 & 0 & 0 & 0 & 0 & 0 \\
\hline
\end{tabular}

the macromolecular class; and in the case of crystalline graphite $\gamma-C$, the symmetry of its crystalline class. The term "macromolecular class", unlike the term "crystalline class", will be used for the symmetry group of equivalent directions in periodic nanostructures with bi- or single-periodic infinite translationinvariant subgroups, whereas the term "crystalline class" describes a point symmetry group of equivalent directions in a three-periodic structure, a crystal, where the translational symmetry elements form an infinite three-periodic subgroup. The representations $\Gamma_{\text {lat. vibr }}$ and $\Gamma_{\pi}$ are determined by the formulas [13]

$\Gamma_{\text {lat. vibr }}=\Gamma_{\text {eq }} \otimes \Gamma_{\text {vector }}, \quad \Gamma_{\pi}=\Gamma_{\text {eq }} \otimes \Gamma_{z}$,

where $\Gamma_{\text {eq }}$ is the atomic equivalence representation at point $\Gamma$, and $\Gamma_{\text {vector }}$ is the representation of the polar vector $\mathbf{r}$ with the components $x, y$, and $z$. While determining $\Gamma_{\pi}$, only $\Gamma_{z}$ is used, which is an irreducible representation for a vector directed along the $z$-axis, because the electron $\pi$-bands in graphene and graphite are formed by electron $p_{z}$-orbitals.

Formulas (14) determine the vibrational and electron representations for elementary excitations with $k \neq 0$ as well [13]. In particular, the character $\chi_{\text {eq }}\left(R_{\alpha}\right)$ of the atomic equivalence representation for the symmetry element $R_{\alpha}$ of the macromolecular and crystalline classes can be defined by the following formula at any point in the Brillouin zone of periodic macromolecular and crystalline structures:

$\chi_{\mathrm{eq}}\left(R_{\alpha}\right)=\sum_{j} \delta_{R_{\alpha} \mathbf{r}_{j}, \mathbf{r}_{j}} e^{i \mathbf{K}_{m} \mathbf{r}_{j}}$.

Here, the operation $R_{\alpha}$ translates an atom into an equivalent position, i.e. it satisfies the condition

$R_{\alpha} \mathbf{r}_{j}=\mathbf{r}_{j}+\mathbf{R}_{n}$

where $\mathbf{R}_{n}=n_{1} \mathbf{a}_{1}+n_{2} \mathbf{a}_{2}+n_{3} \mathbf{a}_{3}\left[\left(n_{1}, n_{2}, n_{3}\right)=\right.$ $=0, \pm 1, \pm 2, \ldots$, and $\mathbf{a}_{i}$ are the basis vectors or the 
Table 10. New classification of projective classes of the group $6 / \mathrm{mmm}\left(\mathrm{D}_{6 \mathrm{~h}}\right)$ and their standard factor-systems: 1 stands for a class $K_{0}, 2-K_{1}, 3-K_{2}, 4-K_{3}, 5-K_{4}, 6-K_{5}, 7-K_{6}, 8-K_{7}$

\begin{tabular}{|c|c|c|c|c|c|}
\hline \multicolumn{6}{|c|}{$K_{0}(\alpha=1, \beta=1, \gamma=1)$} \\
\hline \multicolumn{2}{|c|}{$\omega_{(0)}^{\prime}\left(r_{2}, r_{1}\right)$} & $a$ & $b$ & $c$ & $d$ \\
\hline \multirow{2}{*}{$a$} & $a_{1}$ & 1 & 1 & 1 & 1 \\
\hline & $a_{2}$ & 1 & 1 & 1 & 1 \\
\hline \multirow{2}{*}{$b$} & $b_{1}$ & 1 & 1 & 1 & 1 \\
\hline & $b_{2}$ & 1 & 1 & 1 & 1 \\
\hline \multirow{2}{*}{$c$} & $c_{1}$ & 1 & 1 & 1 & 1 \\
\hline & $c_{2}$ & 1 & 1 & 1 & 1 \\
\hline \multirow{2}{*}{$d$} & $d_{1}$ & 1 & 1 & 1 & 1 \\
\hline & $d_{2}$ & 1 & 1 & 1 & 1 \\
\hline
\end{tabular}

1

$K_{4}(\alpha=1, \beta=1, \gamma=-1)$

\begin{tabular}{r|c|c|c|c|c}
$\omega_{(4)}^{\prime}\left(r_{2}, r_{1}\right)$ & $a$ & $b$ & $c$ & $d$ \\
\hline$a$ & $a_{1}$ & 1 & 1 & 1 & 1 \\
\hdashline & $a_{2}$ & 1 & 1 & 1 & 1 \\
\hdashline$b$ & $b_{1}$ & 1 & 1 & -1 & -1 \\
\hline$-b_{2}$ & 1 & 1 & -1 & -1 \\
\hline$c$ & $c_{1}$ & 1 & 1 & 1 & 1 \\
\hdashline$c_{2}$ & 1 & 1 & 1 & 1 \\
\hdashline & $d_{1}$ & 1 & 1 & -1 & -1 \\
\hdashline$d$ & $d_{2}$ & 1 & 1 & -1 & -1 \\
\hline
\end{tabular}

5
$K_{1}(\alpha=-1, \beta=1, \gamma=1)$

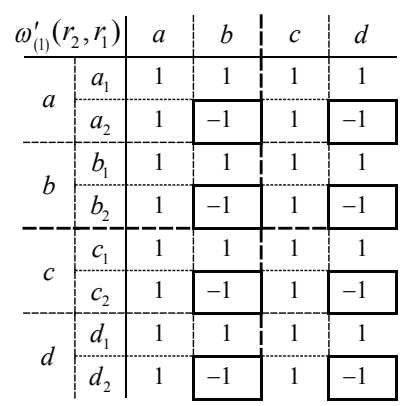

2

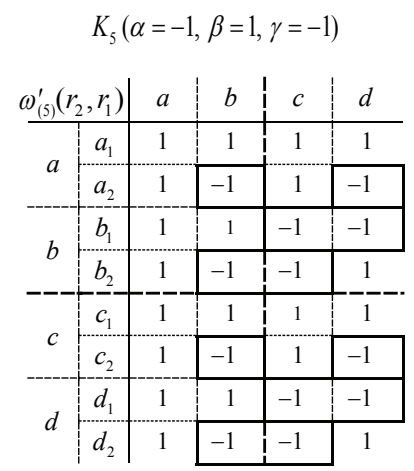

6

lattice translation vectors] is a vector of the crystal lattice or periodic nanostructure, and the condition

$R_{\alpha}^{-1} \mathbf{k}=\mathbf{k}+\mathbf{K}_{m}$

where $\mathbf{K}_{m}=m_{1} \mathbf{b}_{1}+m_{2} \mathbf{b}_{2}+m_{3} \mathbf{b}_{3}\left[\left(m_{1}, m_{2}, m_{3}\right)=\right.$ $=0, \pm 1, \pm 2, \ldots$, and $\mathbf{b}_{j}$ are the basis vectors of reciprocal lattice] is the vector of the reciprocal lattice in the crystal or periodic nanostructure. As usual, it is implied that the basis vectors of the direct and reciprocal lattices are connected by the relations

$\mathbf{b}_{j} \mathbf{a}_{i}=2 \pi \delta_{i j}$.

Let us calculate the distribution of normal vibrations over the symmetry types for various points in the Brillouin zones of single-layer graphene $C_{L 1}$ and crystalline graphite $\gamma-C$. Furthermore, we will determine the symmetry of their electron bands located higher than the valent $\pi$-bands (they will be called the $\pi$-bands), but below the conduction $\pi$-bands (they will be called the $\pi^{*}$-bands).

$K_{2}(\alpha=1, \beta=-1, \gamma=1)$

$K_{3}(\alpha=-1, \beta=-1, \gamma=1)$

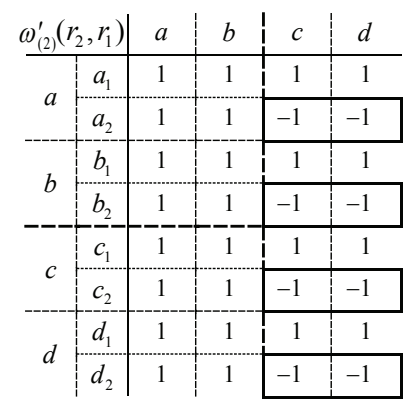

3

$$
K_{6}(\alpha=1, \beta=-1, \gamma=-1)
$$

\begin{tabular}{r|c|c|c|c|c}
$\omega_{(6)}^{\prime}\left(r_{2}, r_{1}\right)$ & $a$ & $b$ & $c$ & $d$ \\
\hline$a$ & $a_{1}$ & 1 & 1 & 1 & 1 \\
\hline & $a_{2}$ & 1 & 1 & -1 & -1 \\
\hdashline$b$ & $b_{1}$ & 1 & 1 & -1 & -1 \\
\hline$b_{2}$ & 1 & 1 & 1 & 1 \\
\hline$c$ & $c_{1}$ & 1 & 1 & 1 & 1 \\
\hline$c_{2}$ & 1 & 1 & -1 & -1 \\
\hline$d$ & $d_{1}$ & 1 & 1 & -1 & -1 \\
\hline & $d_{2}$ & 1 & 1 & 1 & 1
\end{tabular}

7

\subsection{Points $\Gamma$}

It is easy to see that the factor-groups of the wave-vector groups with respect to the translationinvariant subgroups are isomorphic to the same point symmetry group $6 / \mathrm{mmm}\left(D_{6 h}\right)$ for both graphene $C_{L 1}$ and crystalline graphite $\gamma-C$. This is true not only for the wave-vector groups at points $\Gamma$ of graphene and crystalline graphite, but also at all points in the graphene and graphite Brillouin zones that are marked by identical letters. Nevertheless, the characters of the equivalence representation $\Gamma_{\mathrm{eq}}$ in formula (10) are different for the points in the Brillouin zones of graphene and crystalline graphite designated by the same letters, because those objects have different structures.

The characters of representations $\Gamma_{\text {eq }}$ for singlelayer graphene $C_{L 1}$ and crystalline graphite $\gamma-C$ are given in Table 11. Table 11 also includes the characters of the polar-vector representations $\Gamma_{\text {vector }}=\Gamma_{\mathbf{r}}$

ISSN 2071-0186. Ukr. J. Phys. 2018. Vol. 63, No. 5 
Table 11. Characters of equivalence representations of the polar vector (point $\Gamma$ ) and $\Gamma_{\boldsymbol{z}}$ (point $\Gamma$ ) for highsymmetry points in the Brillouin zones of single-layer graphene $C_{L 1}$ and crystalline graphite $\gamma-C$ structures

Points $\Gamma$

Point group(s) 6/mmm $\left(D_{6 h}\right)$

Projective class(es) $K_{0}$

\begin{tabular}{|c|c|c|c|c|c|c|c|c|c|c|c|c|c|c|}
\hline \multicolumn{3}{|c|}{$6 / m m m\left(D_{6 h}\right)$} & $e$ & $2 c_{3}$ & $3 u_{2}$ & $c_{2}$ & $2 c_{6}$ & $3 u_{2}^{\prime}$ & $i$ & $2 i c_{3}$ & $3 i u_{2}$ & $i c_{2}$ & $2 i c_{6}$ & $3 i u_{2}^{\prime}$ \\
\hline $\begin{array}{c}C_{L 1} \\
\gamma-C\end{array}$ & $\begin{array}{l}\mathbf{k}=0 \\
\mathbf{k}=0\end{array}$ & $\begin{array}{c}\Gamma_{\text {eq }} \\
\Gamma_{\text {eq }} \\
\Gamma_{\mathbf{r}} \\
\Gamma_{z}\end{array}$ & $\begin{array}{l}2 \\
4 \\
3 \\
1\end{array}$ & $\begin{array}{l}2 \\
4 \\
0 \\
1\end{array}$ & $\begin{array}{c}0 \\
0 \\
-1 \\
-1\end{array}$ & $\begin{array}{c}0 \\
0 \\
-1 \\
1\end{array}$ & $\begin{array}{l}0 \\
0 \\
2 \\
1\end{array}$ & $\begin{array}{c}2 \\
4 \\
-1 \\
-1\end{array}$ & $\begin{array}{c}0 \\
0 \\
-3 \\
-1\end{array}$ & $\begin{array}{c}0 \\
0 \\
0 \\
-1\end{array}$ & $\begin{array}{l}2 \\
4 \\
1 \\
1\end{array}$ & $\begin{array}{c}2 \\
4 \\
1 \\
-1\end{array}$ & $\begin{array}{c}2 \\
4 \\
-2 \\
-1\end{array}$ & $\begin{array}{l}0 \\
0 \\
1 \\
1\end{array}$ \\
\hline
\end{tabular}

Point $A$

Point group $6 / \mathrm{mmm}\left(D_{6 h}\right)$

Projective class $K_{5}$

\begin{tabular}{|c|c|c|c|c|c|c|c|c|c|c|c|c|}
\hline $6 / m m m\left(D_{6 h}\right)$ & $e$ & $2 c_{3}$ & $3 u_{2}$ & $c_{2}$ & $2 c_{6}$ & $3 u_{2}^{\prime}$ & $i$ & $2 i c_{3}$ & $3 i u_{2}$ & $i c_{2}$ & $2 i c_{6}$ & $3 i u_{2}^{\prime}$ \\
\hline$\gamma-C\left|\mathbf{k}=-(1 / 2) \mathbf{b}_{1}\right| A_{\mathrm{eq}} \mid$ & 4 & 4 & 0 & 0 & 0 & 0 & 0 & 0 & 4 & 0 & 0 & 0 \\
\hline
\end{tabular}

Points $K$

Point groups $\overline{6} m 2\left(D_{3 h}\right)$

Projective classes $K_{0}$

\begin{tabular}{|c|l|c|c|c|c|c|c|}
\hline \multicolumn{2}{|c|}{$\overline{6} m 2\left(D_{3 h}\right)$} & $e$ & $2 c_{3}$ & $3 u_{2}$ & $i c_{2}$ & $2 i c_{6}$ & $3 i u_{2}^{\prime}$ \\
\hline$C_{L 1}$ & $\begin{array}{l}\left(\mathbf{k}_{K}\right)_{1}=-(1 / 3)\left(2 \mathbf{b}_{1}-\mathbf{b}_{2}\right) \\
\left(\mathbf{k}_{K}\right)_{2}=(1 / 3)\left(2 \mathbf{b}_{1}-\mathbf{b}_{2}\right)\end{array}$ & $K_{\text {eq }}$ & 2 & -1 & 0 & 2 \\
$\gamma-C$ & $\begin{array}{l}\left(\mathbf{k}_{K}\right)_{1}=-(1 / 3)\left(2 \mathbf{b}_{2}-\mathbf{b}_{3}\right) \\
\left.\mathbf{k}_{K}\right)_{2}=(1 / 3)\left(2 \mathbf{b}_{2}-\mathbf{b}_{3}\right)\end{array}$ & $K_{\text {eq }}$ & 4 & 1 & 0 & 0 \\
& & & & 0 \\
\end{tabular}

Point $H$

Point group $\overline{6} m 2\left(D_{3 h}\right)$

Projective class $K_{1}$

\begin{tabular}{|c|c|c|c|c|c|c|c|c|c|c|}
\hline \multicolumn{3}{|c|}{$\overline{6} m 2\left(D_{3 h}\right)$} & $e$ & $c_{3}$ & $c_{3}^{2}$ & $3 u_{2}$ & $i c_{2}$ & $i c_{6}^{5}$ & $i c_{6}$ & $3 i u_{2}^{\prime}$ \\
\hline$\gamma-C$ & $\begin{array}{l}\left(\mathbf{k}_{H}\right)_{1}=-(1 / 2) \mathbf{b}_{1}-(1 / 3)\left(2 \mathbf{b}_{2}-\mathbf{b}_{3}\right) \\
\left(\mathbf{k}_{H}\right)_{2}=-(1 / 2) \mathbf{b}_{1}+(1 / 3)\left(2 \mathbf{b}_{2}-\mathbf{b}_{3}\right)\end{array}$ & $H_{\text {eq }}$ & 4 & 1 & 1 & 0 & 0 & $\sqrt{3}$ & $-\sqrt{3}$ & 0 \\
\hline
\end{tabular}

Points $M$

Point groups $m m m\left(D_{2 h}\right)$

Projective classes $K_{0}$

\begin{tabular}{|c|c|c|c|c|c|c|c|c|c|c|}
\hline \multicolumn{3}{|c|}{$m m m\left(D_{2 h}\right)$} & $e$ & $\left(u_{2}\right)_{1}$ & $c_{2}$ & $\left(u_{2}^{\prime}\right)_{1}$ & $i$ & $i\left(u_{2}\right)_{1}$ & $i c_{2}$ & $i\left(u_{2}^{\prime}\right)_{1}$ \\
\hline$C_{L 1}$ & $\begin{array}{l}\left(\mathbf{k}_{M}\right)_{1}=-(1 / 2) \mathbf{b}_{2},\left(\mathbf{k}_{M}\right)_{2}=(1 / 2) \mathbf{b}_{1} \\
\left(\mathbf{k}_{M}\right)_{3}=-(1 / 2)\left(\mathbf{b}_{1}-\mathbf{b}_{2}\right)\end{array}$ & $M_{\text {eq }}$ & 2 & 0 & 0 & 2 & 0 & 2 & 2 & 0 \\
\hline$\gamma-C$ & $\begin{array}{l}\left(\mathbf{k}_{M}\right)_{1}=-(1 / 2) \mathbf{b}_{3},\left(\mathbf{k}_{M}\right)_{2}=(1 / 2) \mathbf{b}_{2} \\
\left(\mathbf{k}_{M}\right)_{3}=-(1 / 2)\left(\mathbf{b}_{2}-\mathbf{b}_{3}\right)\end{array}$ & $M_{\text {eq }}$ & 4 & 0 & 0 & 4 & 0 & 4 & 4 & 0 \\
\hline
\end{tabular}

Point $L$

Point group $m m m\left(D_{2 h}\right)$

Projective class $K_{5}$

\begin{tabular}{|c|l|c|c|c|c|c|c|c|c|}
\hline \multicolumn{2}{|c|}{$m m m\left(D_{2 h}\right)$} & $e$ & $\left(u_{2}\right)_{1}$ & $c_{2}$ & $\left(u_{2}^{\prime}\right)_{1}$ & $i$ & $i\left(u_{2}\right)_{1}$ & $i c_{2}$ & $i\left(u_{2}^{\prime}\right)_{1}$ \\
\hline$\gamma-C$ & $\begin{array}{l}\left(\mathbf{k}_{L}\right)_{1}=-(1 / 2)\left(\mathbf{b}_{1}+\mathbf{b}_{3}\right) \\
\left(\mathbf{k}_{L}\right)_{2}=-(1 / 2)\left(\mathbf{b}_{1}-\mathbf{b}_{2}\right) \\
\left(\mathbf{k}_{L}\right)_{3}=-(1 / 2)\left(\mathbf{b}_{1}+\mathbf{b}_{2}-\mathbf{b}_{3}\right)\end{array}$ & $L_{\text {eq }}$ & 4 & 0 & 0 & 0 & 0 & 4 & 0 \\
\end{tabular}

ISSN 2071-0186. Ukr. J. Phys. 2018. Vol. 63, No. 5 
Table 12. Distributions of vibrational and electron excitations for the $\pi$-bands at high-symmetry points in the Brillouin zones over irreducible projective representations of the corresponding projective classes for single-layer graphene $C_{L 1}$ and crystalline graphite $\gamma-C$ structures

\begin{tabular}{|c|c|}
\hline $\begin{array}{c}\text { Single-layer } \\
\text { graphene, } C_{L 1}\end{array}$ & $\begin{array}{c}\text { Crystalline } \\
\text { graphite, } \gamma-C\end{array}$ \\
\hline \multicolumn{2}{|c|}{$\begin{array}{l}\text { Points } \Gamma \\
\text { int groups } 6 / m m m\left(D_{6 h}\right) \\
\text { Projective classes } K_{0}\end{array}$} \\
\hline 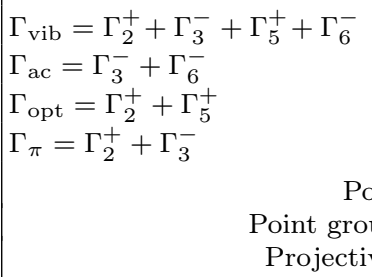 & $\begin{array}{l}\Gamma_{\mathrm{vib}}=2 \Gamma_{2}^{+}+2 \Gamma_{3}^{-}+2 \Gamma_{5}^{+}+2 \Gamma_{6}^{-} \\
\Gamma_{\mathrm{ac}}=\Gamma_{3}^{-}+\Gamma_{6}^{-} \\
\Gamma_{\mathrm{opt}}=2 \Gamma_{2}^{+}+\Gamma_{3}^{-}+2 \Gamma_{5}^{+}+\Gamma_{6}^{-} \\
\Gamma_{\pi}=2 \Gamma_{2}^{+}+2 \Gamma_{3}^{-} \\
\text {ints } K \\
\text { ips } \overline{6} m 2\left(D_{3 h}\right) \\
\text { e classes } K_{0}\end{array}$ \\
\hline $\begin{array}{l}K_{\mathrm{vib}}=K_{1}+K_{3}+K_{5}+K_{6} \\
K_{\pi}=K_{6}\end{array}$ & $\mid \begin{array}{l}K_{\mathrm{vib}}=K_{1}+K_{2}+K_{3}+ \\
+K_{4}+3 K_{5}+K_{6} \\
K_{\pi}=K_{2}+K_{4}+K_{6}\end{array}$ \\
\hline \multicolumn{2}{|c|}{$\begin{array}{c}\text { Points } M \\
\text { Point groups } m m m\left(D_{2 h}\right) \\
\text { Projective classes } K_{0}\end{array}$} \\
\hline $\begin{array}{l}M_{\mathrm{vib}}=M_{1}^{+}+M_{2}^{+}+M_{2}^{-}+ \\
+M_{3}^{+}+M_{3}^{-}+M_{4}^{-} \\
M_{\pi}=M_{2}^{+}+M_{3}^{-}\end{array}$ & $\mid \begin{array}{l}M_{\mathrm{vib}}=2 M_{1}^{+}+2 M_{2}^{+}+2 M_{2}^{-}+ \\
+2 M_{3}^{+}+2 M_{3}^{-}+2 M_{4}^{-} \\
M_{\pi}=2 M_{2}^{+}+2 M_{3}^{-}\end{array}$ \\
\hline \multicolumn{2}{|c|}{$\begin{array}{c}\text { Point } A \\
\begin{array}{r}\text { Point group } 6 / \mathrm{mmm}\left(D_{6 h}\right) \\
\text { Projective class } K_{5}\end{array} \\
\mid \begin{array}{l}A_{\mathrm{vib}}=2 A_{1}^{(5)}+2 A_{3}^{(5)} \\
A_{\pi}=2 A_{1}^{(5)}\end{array}\end{array}$} \\
\hline \multicolumn{2}{|c|}{$\begin{array}{c}\text { Point } H \\
\text { Point group } \overline{6} m 2\left(D_{3 h}\right) \\
\text { Projective class } K_{1}\end{array}$} \\
\hline 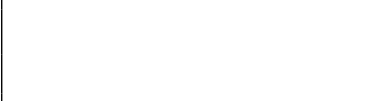 & $\mid \begin{array}{l}H_{\mathrm{vib}}=3 H_{1}^{(1)}+H_{2}^{(1)}+2 H_{3}^{(1)} \\
H_{\pi}=H_{1}^{(1)}+H_{3}^{(1)}\end{array}$ \\
\hline $\begin{array}{r}\mathrm{P} \\
\text { Point grou } \\
\text { Project }\end{array}$ & $\begin{array}{l}\text { p } m m m\left(D_{2 h}\right) \\
\text { ve class } K_{5} \\
L_{\mathrm{vib}}=4 L_{1}^{(5)}+2 L_{2}^{(5)} \\
L_{\pi}=2 L_{1}^{(5)}\end{array}$ \\
\hline
\end{tabular}

and the representation $\Gamma_{z}$ that determines the symmetry of the $p_{z}$-orbital. Table 12 illustrates the distribution of the vibrational representation $\Gamma_{\text {vibr }} \equiv$ $\equiv \Gamma_{\text {lat. vibr }}$ [it can easily be obtained from formula (10)], the representations for the acoustic, $\Gamma_{a c}$, and optical, $\Gamma_{\mathrm{opt}}$, fundamental vibrational modes, and the
Table 13. Characters of single- and two-valued irreducible projective representations of point $\Delta$

\begin{tabular}{|c|c|c|c|c|c|c|c|c|c|}
\hline \multirow{2}{*}{$\begin{array}{c}\text { Projec- } \\
\text { tive } \\
\text { class }\end{array}$} & \multirow{2}{*}{$\begin{array}{l}\text { Indication } \\
\quad \text { of an } \\
\text { irreducible } \\
\text { projective } \\
\text { represen- } \\
\text { tation }\end{array}$} & \multicolumn{8}{|c|}{$6 / m m m\left(D_{6 h}\right)$} \\
\hline & & $e$ & $c_{3}$ & $c_{3}^{2}$ & $3 \sigma_{r}^{\prime}$ & $c_{2}$ & $c_{6}^{5}$ & $c_{6}$ & $3 \sigma_{r}$ \\
\hline \multirow[t]{6}{*}{$K_{0}$} & $\Delta_{1}$ & 1 & 1 & 1 & $\eta_{k}$ & $\eta_{k}$ & $\eta_{k}$ & $\eta_{k}$ & 1 \\
\hline & $\Delta_{2}$ & 1 & 1 & 1 & $\eta_{k}$ & $-\eta_{k}$ & $-\eta_{k}$ & $-\eta_{k}$ & -1 \\
\hline & $\Delta_{3}$ & 1 & 1 & 1 & $-\eta_{k}$ & $\eta_{k}$ & $\eta_{k}$ & $\eta_{k}$ & -1 \\
\hline & $\Delta_{4}$ & 1 & 1 & 1 & $-\eta_{k}$ & $-\eta_{k}$ & $-\eta_{k}$ & $-\eta_{k}$ & 1 \\
\hline & $\Delta_{5}$ & 2 & -2 & -1 & 0 & $2 \eta_{k}$ & $-\eta_{k}$ & $-\eta_{k}$ & 0 \\
\hline & $\Delta_{6}$ & 2 & -1 & -1 & 0 & $-2 \eta_{k}$ & $\eta_{k}$ & $\eta_{k}$ & 0 \\
\hline \multirow[t]{3}{*}{$K_{1}$} & $\Delta_{7}$ & 2 & 1 & -1 & 0 & 0 & $\sqrt{3} \eta_{k}$ & $-\sqrt{3} \eta_{k}$ & 0 \\
\hline & $\Delta_{8}$ & 2 & 1 & -1 & 0 & & $-\sqrt{3} \eta_{k}$ & $\sqrt{3} \eta_{k}$ & 0 \\
\hline & $\Delta_{9}$ & 2 & -2 & 2 & 0 & 0 & 0 & 0 & 0 \\
\hline
\end{tabular}

electron-band representation $\Gamma_{\pi}$ [it was also found from formula (10)] over the irreducible representations of the group $6 / m m m\left(D_{6 h}\right)$.

\subsection{Point A}

The factor-group of the wave-vector group at point $A$ for graphite crystals $\gamma-C$ is also isomorphic to the group $6 / m m m\left(D_{6 h}\right)$ with respect to the translationinvariant subgroup. The wave-vector star at point $A$, as was at point $\Gamma$, consists of a single vector $\mathbf{k}=-(1 / 2) \mathbf{b}_{1}$. The character of the projective equivalence representation at point $A$, i.e. the representation $A_{\text {eq }}$, is given in Table 11 , and the distributions of the representations $A_{\text {vibr }}$ and $A_{\pi}$ over the irreducible group representations in Table 12 .

Table 13 illustrates the characters of one- and two-valued irreducible projective representations of point $\Delta$ located between points $\Gamma$ and $A$. The group of equivalent directions of the wave-vector group at point $\Delta$ is the group $6 m m\left(C_{6 v}\right)$. The wave-vector star at this point contains two rays. There is no additional degeneration of states, if their invariance with respect to time inversion is taken into account. This is in contrast to the pairwise merging of dispersion curves for all energy states at point $A$ owing to the invariance with respect to the time inversion for structures, whose symmetry is described by the non-symmetric spatial group $P 6_{3} / m m c$ (this behavior can be derived on the basis of a similar consideration for the wurzite structure made in work [6]).

ISSN 2071-0186. Ukr. J. Phys. 2018. Vol. 63, No. 5 
Figure 5 exhibits a diagram that can be used to determine the compatibility of irreducible projective representations of the group $P 6_{3} / m m c$ along the line $\Gamma-\Delta-A$ in the Brillouin zone of crystalline graphite.

\subsection{Points $K$}

The factor-groups of the wave-vector groups with respect to infinite translation-invariant subgroups at points $K$ of single-layer graphene $C_{L 1}$ and crystalline graphite $\gamma-C$ structures are isomorphic to the point group $\overline{6} m 2\left(D_{3 h}\right)$, which is a point symmetry group of equivalent directions. The stars of the wave-vector groups at points $K$ in both structures contain two vectors: for single-layer graphene $C_{L 1}$, these are $\left(\mathbf{k}_{K}\right)_{1}=-(1 / 3)\left(2 \mathbf{b}_{1}-\mathbf{b}_{2}\right)$ and $\left(\mathbf{k}_{K}\right)_{2}=$ $=(1 / 3)\left(2 \mathbf{b}_{1}-\mathbf{b}_{2}\right)$; and for crystalline graphite $\gamma-C$, these are $\left(\mathbf{k}_{K}\right)_{1}=-(1 / 3)\left(2 \mathbf{b}_{2}-\mathbf{b}_{3}\right)$ and $\left(\mathbf{k}_{K}\right)_{2}=$ $=(1 / 3)\left(2 \mathbf{b}_{2}-\mathbf{b}_{3}\right)$. The characters of the projective representations $K_{\text {eq }}$ are given in Table 11 (for both structures, they belong to the class $K_{0}$ ), and the distributions $K_{\text {vibr }}$ and $K_{\pi}$ over the irreducible representations of the group $\overline{6} m 2\left(D_{3 h}\right)$ are presented in Table 12 .

\subsection{Point $\mathrm{H}$}

The factor-group of the wave-vector group with respect to the infinite translation-invariant subgroup at point $H$ of the graphite $\gamma-C$ structure is also isomorphic to the point group $\overline{6} m 2\left(D_{3 h}\right)$. The star of the wave-vector group consists of two vectors: $\left(\mathbf{k}_{H}\right)_{1}=-(1 / 2) \mathbf{b}_{1}-(1 / 3)\left(2 \mathbf{b}_{2}-\mathbf{b}_{3}\right)$ and $\left(\mathbf{k}_{H}\right)_{2}=$ $=-(1 / 2) \mathbf{b}_{1}+(1 / 3)\left(2 \mathbf{b}_{2}-\mathbf{b}_{3}\right)$. Table $14, a$ presents the factor system $\omega_{1, H}\left(r_{2}, r_{1}\right)$ calculated by formula (8). With the help of the function values on the group $u_{1, H}(r)$, which are indicated in the lower part of Table 14, $a$, it is reduced to the standard form $\omega_{1, H}^{\prime}\left(r_{2}, r_{1}\right)$, which is represented in Table $14, b$. It is easy to see that the factor systems $\omega_{1, H}\left(r_{2}, r_{1}\right)$ and $\omega_{1, H}^{\prime}\left(r_{2}, r_{1}\right)$-in accordance with the values of the coefficients $\alpha, \beta$, and $\gamma$, which are determined directly from the factor-systems themselves-belong to the projective class $K_{1}$, i.e. $\omega_{1, H}^{\prime}\left(r_{2}, r_{1}\right) \equiv \omega_{(1)}^{\prime}\left(r_{2}, r_{1}\right)$.

Besides the factor-system $\omega_{1, H}^{\prime}\left(r_{2}, r_{1}\right)$ describing the symmetric properties of the spatial symmetry group $P 6_{3} / m m c\left(D_{6 h}^{4}\right)$ of crystalline graphite, the standard factor-system of the projective class $K_{1}$, i.e. the factor-system $\omega_{(1)}^{\prime}\left(r_{2}, r_{1}\right)$, can also be obtained from the factor system $\omega_{2}\left(r_{2}, r_{1}\right)$. The latter is determined by the spinor transformations un-

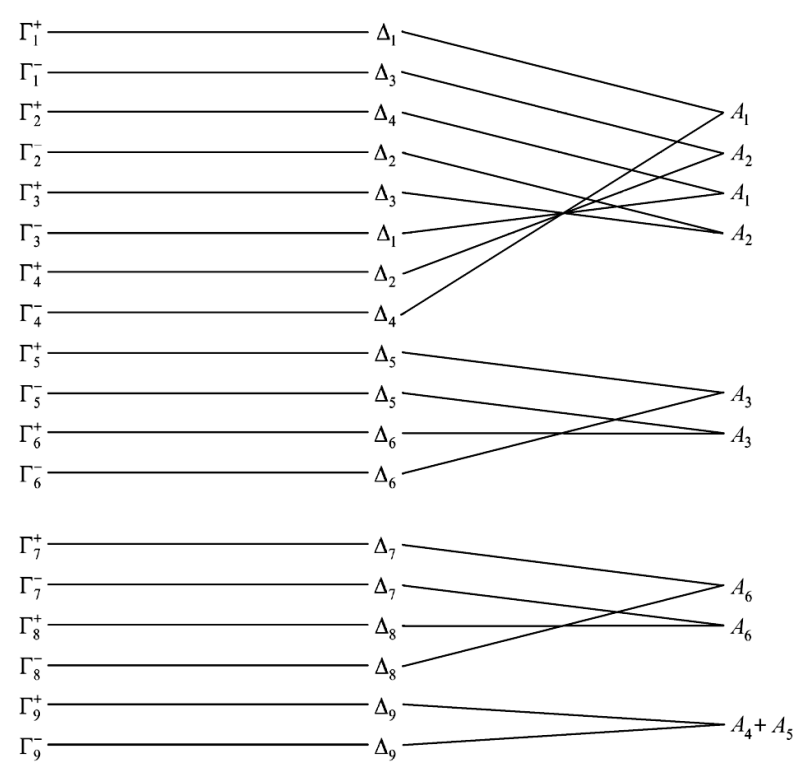

Fig. 5. Diagram determining the compatibility of irreducible projective representations of the $P 6_{3} / m m c$ group in the Brillouin zone in the $\Gamma-A$ direction

der the action of the symmetry elements belonging to the point group $\overline{6} m 2\left(D_{3 h}\right)$, which is a symmetry group of equivalent directions in the wavevector group of point $H$ of the Brillouin zone of graphite. In Table 15, $a$, the factor-system $\omega_{2}\left(r_{2}, r_{1}\right)$ for the point symmetry group $\overline{6} m 2\left(D_{3 h}\right)$ is presented, which was obtained by formula (12). One can easily see that it belongs to the projective class $K_{1}$. As was done above, the parenthesized subscripts display the multiplication table for the elements of the group $\overline{6} m 2$ (the numbers in parentheses indicate the numerical designation of elements for the group $\overline{6} m 2$, which correspond to the products $\left.r_{2} r_{1}\right)$. Table $15, b$ contains the corresponding standard factor-system $\omega_{2}^{\prime}\left(r_{2}, r_{1}\right)$. It was obtained by transforming the factor-system $\omega_{2}\left(r_{2}, r_{1}\right)$ by means of formula (5) and using the values of the function $u_{2}(r)$ that are indicated in the lower part of Table 15, $a$.

It is important that the values of the coefficients $u_{2}(r)$ for the elements entering the both groups $\overline{6} \mathrm{~m} 2$ and $6 / \mathrm{mmm}$ turned out identical. The standard factor-systems $\omega_{1, H}^{\prime}\left(r_{2}, r_{1}\right)$ and $\omega_{2}^{\prime}\left(r_{2}, r_{1}\right)$ belonging to the same projective class $K_{1}$ also expectedly coincide; i.e. the equality $\omega_{1, H}^{\prime}\left(r_{2}, r_{1}\right)=\omega_{2}^{\prime}\left(r_{2}, r_{1}\right)=$ $=\omega_{(1)}^{\prime}\left(r_{2}, r_{1}\right)$ does take place.

The characters of various $p$-equivalent forms of irreducible projective representations of the group 
Table 14. Factor-systems $\boldsymbol{\omega}_{1, H}\left(\boldsymbol{r}_{\mathbf{2}}, \boldsymbol{r}_{1}\right)$ for point $\boldsymbol{H}$ in crystalline

graphite (the spatial group $P 6_{3} / m m c\left(D_{6 h}^{4}\right)$, point group $\left.\overline{6} m 2\left(D_{3 h}\right)\right)(a)$ and the standard factor-system $\omega_{1, H}^{\prime}\left(r_{2}, r_{1}\right)$ corresponding to its standard form $(b)$. The lower part of Table 14, $a$ contains the values of the function $u_{1, H}(r)$ that transform the factor-system $\omega_{1, H}\left(r_{2}, r_{1}\right)$ to the standard form $\omega_{1, H}^{\prime}\left(r_{2}, r_{1}\right) \equiv \omega_{(1)}^{\prime}\left(r_{2}, r_{1}\right)$

\begin{tabular}{|c|c|c|c|c|c|c|c|c|c|c|c|c|c|}
\hline$\omega_{1, H}\left(r_{2}, r_{1}\right)$ & ${ }_{r_{2}} r_{1}$ & $\begin{array}{l}1 \\
e\end{array}$ & $\begin{array}{l}2 \\
c_{3}\end{array}$ & $\begin{array}{c}3 \\
c_{3}^{2} \\
\end{array}$ & $\begin{array}{c}4 \\
\left(u_{2}\right)_{1}\end{array}$ & $\begin{array}{c}5 \\
\left(u_{2}\right)_{2}\end{array}$ & $\begin{array}{c}6 \\
\left(u_{2}\right)_{3}\end{array}$ & $\begin{array}{c}7 \\
i c_{2}\end{array}$ & $\begin{array}{c}8 \\
i c_{6}^{5}\end{array}$ & $\begin{array}{c}9 \\
i c_{6}\end{array}$ & $\begin{array}{c}10 \\
i\left(u_{2}^{\prime}\right)_{1}\end{array}$ & $\begin{array}{c}11 \\
i\left(u_{2}^{\prime}\right)_{2}\end{array}$ & $\begin{array}{c}12 \\
i\left(u_{2}^{\prime}\right)_{3}\end{array}$ \\
\hline 1 & $e$ & $1_{(1)}$ & $1_{(2)}$ & $1_{(3)}$ & $1_{(4)}$ & $1_{(5)}$ & $1_{(6)}$ & $1_{(7)}$ & $\mathrm{I}_{(8)}$ & $\mathbf{I}_{(9)}$ & $I_{(10)}$ & $I_{(11)}$ & $1_{(12)}$ \\
\hline 2 & $c_{3}$ & $1_{(2)}$ & $1_{(3)}$ & $1_{(1)}$ & $1_{(6)}$ & $1_{(4)}$ & $1_{(5)}$ & $I_{(8)}$ & $1_{(9)}$ & $1_{(7)}$ & $1_{(12)}$ & $1_{(10)}$ & $1_{(11)}$ \\
\hline 3 & $c_{3}^{2}$ & $1_{(3)}$ & $1_{(1)}$ & $\mathbf{I}_{(2)}$ & $1_{(5)}$ & $I_{(6)}$ & $1_{(4)}$ & $1_{(9)}$ & $l_{(7)}$ & $1_{(8)}$ & $1_{(11)}$ & $1_{(12)}$ & $1_{(10)}$ \\
\hline 4 & $\left(u_{2}\right)_{1}$ & $1_{(4)}$ & $1_{(5)}$ & $1_{(6)}$ & $1_{(1)}$ & $\mathrm{I}_{(2)}$ & $1_{(3)}$ & $-1_{(10)}$ & $-1_{(11)}$ & $-1_{(12)}$ & $-1_{(7)}$ & $-1_{(8)}$ & $-1_{(9)}$ \\
\hline 5 & $\left(u_{2}\right)_{2}$ & $I_{(5)}$ & $1_{(6)}$ & $1_{(4)}$ & $1_{(3)}$ & $1_{(1)}$ & $1_{(2)}$ & $-1_{(11)}$ & $-1_{(12)}$ & $-1_{(10)}$ & $-1_{(9)}$ & $-1_{(7)}$ & $-1_{(8)}$ \\
\hline 6 & $\left(u_{2}\right)_{3}$ & $1_{(6)}$ & $\mathrm{I}_{(4)}$ & $I_{(5)}$ & $1_{(2)}$ & $1_{(3)}$ & $1_{(1)}$ & $-1_{(12)}$ & $-1_{(10)}$ & $-1_{(11)}$ & $-1_{(8)}$ & $-1_{(9)}$ & $-1_{(7)}$ \\
\hline 7 & $i c_{2}$ & $1_{(7)}$ & $1_{(8)}$ & $1_{(9)}$ & $1_{(10)}$ & $1_{(11)}$ & $1_{(12)}$ & $-1_{(1)}$ & $-1_{(2)}$ & $-1_{(3)}$ & $-1_{(4)}$ & $-1_{(5)}$ & $-1_{(6)}$ \\
\hline 8 & $i c_{6}^{5}$ & $1_{(8)}$ & $1_{(9)}$ & $1_{(7)}$ & $1_{(12)}$ & $I_{(10)}$ & $1_{(11)}$ & $-1_{(2)}$ & $-1_{(3)}$ & $-1_{(1)}$ & $-1_{(6)}$ & $-1_{(4)}$ & $-1_{(5)}$ \\
\hline 9 & $i c_{6}$ & $I_{(9)}$ & $1_{(7)}$ & $1_{(8)}$ & $1_{(11)}$ & $1_{(12)}$ & $1_{(10)}$ & $-1_{(3)}$ & $-1_{(1)}$ & $-1_{(2)}$ & $-1_{(5)}$ & $-1_{(6)}$ & $-1_{(4)}$ \\
\hline 10 & $i\left(u_{2}^{\prime}\right)_{1}$ & $1_{(10)}$ & $1_{(11)}$ & $1_{(12)}$ & $1_{(7)}$ & $\mathrm{l}_{(8)}$ & $I_{(9)}$ & $l_{(4)}$ & $l_{(5)}$ & $I_{(6)}$ & $1_{(1)}$ & $1_{(2)}$ & $1_{(3)}$ \\
\hline 11 & $i\left(u_{2}^{\prime}\right)_{2}$ & $1_{(11)}$ & $1_{(12)}$ & $1_{(10)}$ & $1_{(9)}$ & $1_{(7)}$ & $1_{(8)}$ & $1_{(5)}$ & $l_{(6)}$ & $I_{(4)}$ & $1_{(3)}$ & $I_{(1)}$ & $1_{(2)}$ \\
\hline 12 & $i\left(u_{2}^{\prime}\right)_{3}$ & $1_{(12)}$ & $1_{(10)}$ & $1_{(11)}$ & $1_{(8)}$ & $1_{(9)}$ & $1_{(7)}$ & $1_{(6)}$ & $1_{(4)}$ & $1_{(5)}$ & $I_{(2)}$ & $l_{(3)}$ & $1_{(1)}$ \\
\hline & $u_{1, H}(r)$ & 1 & 1 & 1 & 1 & 1 & 1 & $i$ & $i$ & $i$ & $i$ & $i$ & $i$ \\
\hline
\end{tabular}

$a$

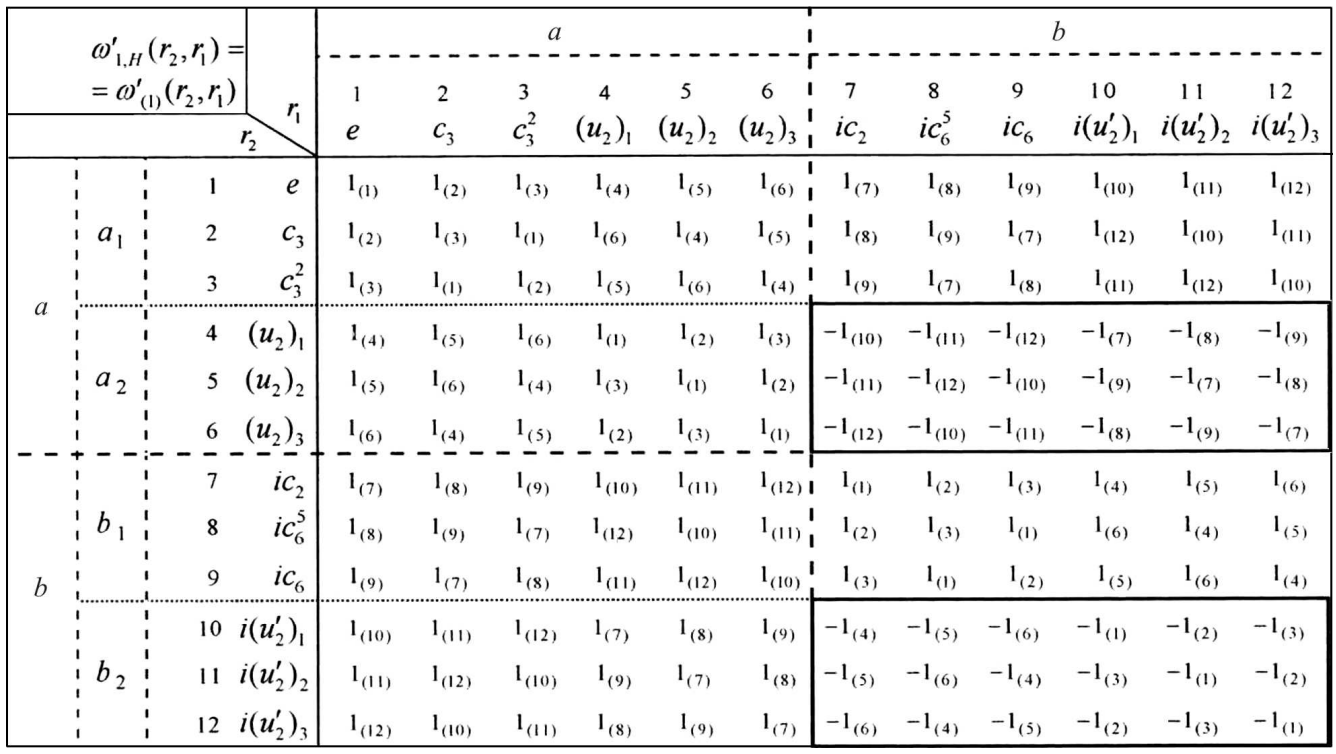

$b$

$\overline{6} m 2\left(D_{3 h}\right)$, which belong to the projective class $K_{1}$, are given in Table 16; in particular, for irreducible single-valued projective representations corresponding to the standard factor-system $\omega_{(1)}^{\prime}\left(r_{2}, r_{1}\right)$ (Table $16, a)$, for irreducible projective two-valued spinor representations (Table 16, $b$ ), and for irreducible projective single-valued representations describing the symmetry of vibrational and electron excitations without making allowance for the spin at point $H$ for the spatial symmetry group or the wave-vector group 
Table 15. Factor-system $\omega_{2}\left(r_{2}, r_{1}\right)$ for the group $\overline{6} m \mathbf{2}\left(D_{3 h}\right)(a)$ and the standard factor-system $\omega_{2}^{\prime}\left(r_{2}, r_{1}\right)$ corresponding to its standard form $(b)$. In the lower part of Table 15, $a$, we present the values of the functions $u_{2}(r)$ that reduce the factor-system $\omega_{2}\left(r_{2}, r_{1}\right)$ to the standard form $\omega_{2}^{\prime}\left(r_{2}, r_{1}\right) \equiv \omega_{(1)}^{\prime}\left(r_{2}, r_{1}\right)$

\begin{tabular}{|c|c|c|c|c|c|c|c|c|c|c|c|c|c|}
\hline$\omega_{2}\left(r_{2}, r_{1}\right)$ & $r_{r_{2}} r_{1}$ & $\begin{array}{l}1 \\
e\end{array}$ & $\begin{array}{l}2 \\
c_{3}\end{array}$ & $\begin{array}{l}3 \\
c_{3}^{2}\end{array}$ & $\begin{array}{c}4 \\
\left(u_{2}\right)_{1}\end{array}$ & $\begin{array}{c}5 \\
\left(u_{2}\right)_{2}\end{array}$ & $\begin{array}{c}6 \\
\left(u_{2}\right)_{3}\end{array}$ & $\begin{array}{c}7 \\
i c_{2}\end{array}$ & $\begin{array}{c}8 \\
i c_{6}^{5}\end{array}$ & $\begin{array}{c}9 \\
i c_{6}\end{array}$ & $\begin{array}{c}10 \\
i\left(u_{2}^{\prime}\right)_{1}\end{array}$ & $\begin{array}{c}11 \\
i\left(u_{2}^{\prime}\right)_{2}\end{array}$ & $\begin{array}{c}12 \\
i\left(u_{2}^{\prime}\right)_{3}\end{array}$ \\
\hline 1 & $e$ & $1_{(1)}$ & $1_{(2)}$ & $1_{(3)}$ & $1_{(4)}$ & $I_{(5)}$ & $1_{(6)}$ & $1_{(7)}$ & $1_{(8)}$ & $\mathbf{1}_{(9)}$ & $1_{(10)}$ & $1_{(11)}$ & $1_{(12)}$ \\
\hline 2 & $c_{3}$ & $\mathrm{I}_{(2)}$ & $1_{(3)}$ & $-1_{(1)}$ & $-1_{(6)}$ & $-1_{(4)}$ & $-1_{(5)}$ & $1_{(8)}$ & $-1_{(9)}$ & $1_{(7)}$ & $-1_{(12)}$ & $-1_{(10)}$ & $-1_{(11)}$ \\
\hline 3 & $c_{3}^{2}$ & $1_{(3)}$ & $-1_{(1)}$ & $-1_{(2)}$ & $1_{(5)}$ & $1_{(6)}$ & $1_{(4)}$ & $-1_{(9)}$ & $-1_{(7)}$ & $1_{(8)}$ & $1_{(11)}$ & $l_{(12)}$ & $1_{(10)}$ \\
\hline 4 & $\left(u_{2}\right)_{1}$ & $1_{(4)}$ & $-1_{(5)}$ & $1_{(6)}$ & $-1_{(1)}$ & $1_{(2)}$ & $-1_{(3)}$ & $-1_{(10)}$ & $1_{(11)}$ & $I_{(12)}$ & $1_{(7)}$ & $-1_{(8)}$ & $-1_{(9)}$ \\
\hline 5 & $\left(u_{2}\right)_{2}$ & $1_{(5)}$ & $-1_{(6)}$ & $1_{(4)}$ & $-1_{(3)}$ & $-1_{(1)}$ & $1_{(2)}$ & $-1_{(11)}$ & $1_{(12)}$ & $l_{(10)}$ & $-1_{(9)}$ & $1_{(7)}$ & $-1_{(8)}$ \\
\hline 6 & $\left(u_{2}\right)_{3}$ & $l_{(6)}$ & $-1_{(4)}$ & $1_{(5)}$ & $\mathrm{I}_{(2)}$ & $-1_{(3)}$ & $-1_{(1)}$ & $-1_{(12)}$ & $\mathrm{I}_{(10)}$ & $\mathrm{I}_{(11)}$ & $-1_{(8)}$ & $-1_{(9)}$ & $1_{(7)}$ \\
\hline 7 & $i c_{2}$ & $1_{(7)}$ & $1_{(8)}$ & $-1_{(9)}$ & $I_{(10)}$ & $1_{(11)}$ & $I_{(12)}$ & $-I_{(1)}$ & $-1_{(2)}$ & $l_{(3)}$ & $-1_{(4)}$ & $-1_{(5)}$ & $-1_{(6)}$ \\
\hline 8 & $i c_{6}^{5}$ & $1_{(8)}$ & $-1_{(9)}$ & $-1_{(7)}$ & $-1_{(12)}$ & $-1_{(10)}$ & $-1_{(11)}$ & $-1_{(2)}$ & $-1_{(3)}$ & $-1_{(1)}$ & $1_{(6)}$ & $1_{(4)}$ & I(5) \\
\hline 9 & $i c_{6}$ & $1_{(9)}$ & $1_{(7)}$ & $1_{(8)}$ & $-1_{(11)}$ & $-1_{(12)}$ & $-1_{(10)}$ & $1_{(3)}$ & $-1_{(1)}$ & $1_{(2)}$ & $1_{(5)}$ & $1_{(6)}$ & $1_{(4)}$ \\
\hline 10 & $i\left(u_{2}^{\prime}\right)_{1}$ & $1_{(10)}$ & $-1_{(11)}$ & $1_{(12)}$ & $-1_{(7)}$ & $1_{(8)}$ & $1_{(9)}$ & $1_{(4)}$ & $-1_{(5)}$ & $-1_{(6)}$ & $-1_{(1)}$ & $I_{(2)}$ & $-1_{(3)}$ \\
\hline 11 & $i\left(u_{2}^{\prime}\right)_{2}$ & $1_{(11)}$ & $-1_{(12)}$ & $I_{(10)}$ & $1_{(9)}$ & $-1_{(7)}$ & $1_{(8)}$ & $I_{(5)}$ & $-1_{(6)}$ & $-1_{(4)}$ & $-1_{(3)}$ & $-1_{(1)}$ & $1_{(2)}$ \\
\hline 12 & $i\left(u_{2}^{\prime}\right)_{3}$ & $1_{(12)}$ & $-1_{(10)}$ & $I_{(11)}$ & $1_{(8)}$ & $1_{(9)}$ & $-1_{(7)}$ & $1_{(6)}$ & $-1_{(4)}$ & $-1_{(5)}$ & $I_{(2)}$ & $-1_{(3)}$ & $-1_{(1)}$ \\
\hline & $u_{2}(r)$ & 1 & -1 & 1 & $i$ & $i$ & $i$ & $i$ & $-i$ & $-i$ & -1 & -1 & -1 \\
\hline
\end{tabular}

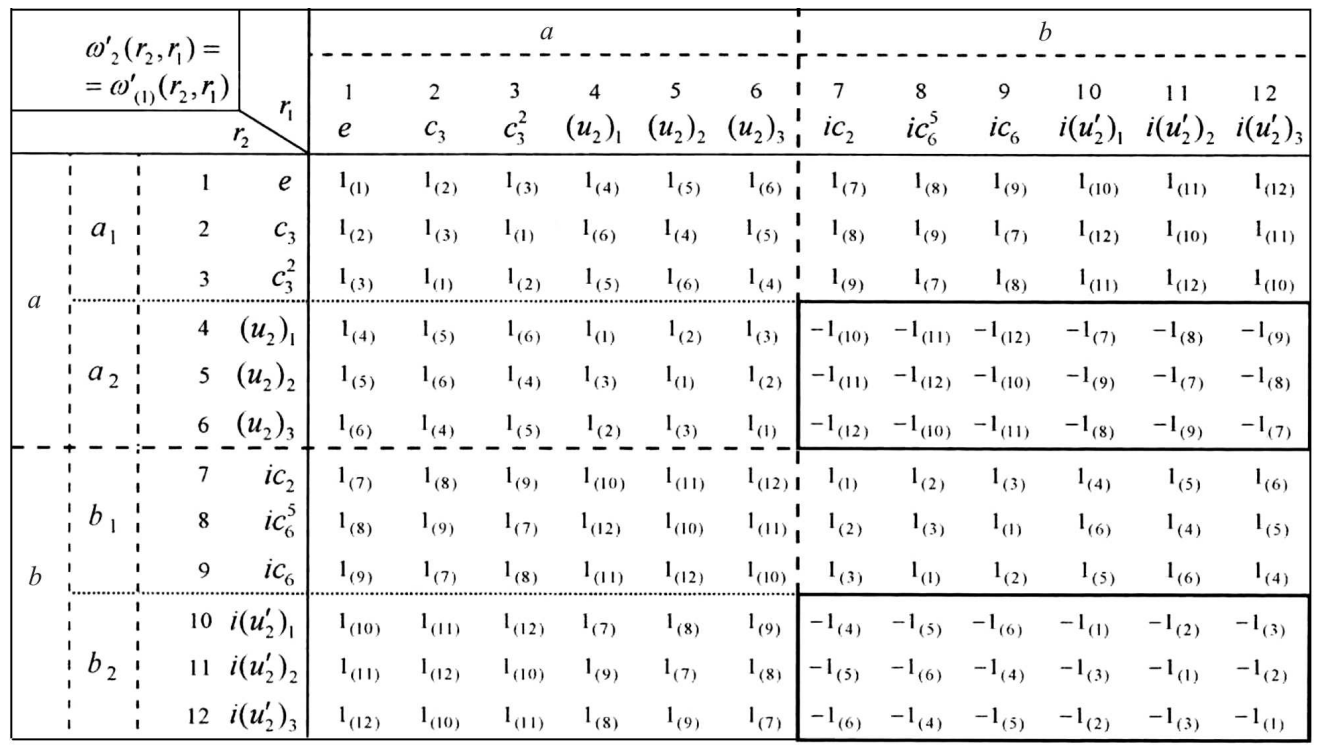

of point $H$, which is a subgroup of the space symmetry group $P 6_{3} / m m c\left(D_{6 h}^{4}\right)$ (Table $\left.16, c\right)$. It should be noted that the representations of the spatial symmetry group $P 6_{3} / m m c$ at point $H$, which were calculated making no allowance for the spin of a quantum excitation, are drastically different from the double- valued spinor representations (Table $16, b)$. But they are projectively equivalent ( $p$-equivalent) to this class, as well as to the class of projective representations corresponding to the standard factor-system of the projective class $K_{1}$ (Table 16,a). Moreover, the "carriers" of the two-valued character of representa- 
Table 16. Characters of irreducible projective representations of the group $\overline{6} m 2\left(D_{3 h}\right)$ : single-valued representations corresponding to the standard factor-system (a), two-valued spinor representations $(b)$, and single-valued representations describing the symmetry of vibrational and electron excitations neglecting the spin at point $H$ for the spatial symmetry group of (the wave-vector group of point $H$ ) that is a subgroup of the spatial symmetry group $\mathrm{Pb}_{3} m m c\left(D_{6 h}\right)(c)$. Primes above letters $\Gamma$ in the symbols of irreducible projective representations mean that those representations are two-valued spinor ones

\begin{tabular}{|c|c|c|c|c|c|c|c|c|c|c|}
\hline \multicolumn{3}{|c|}{$\overline{6} m 2\left(D_{3 h}\right)$} & $e$ & $c_{3}$ & $c_{3}^{2}$ & $3 i u_{2}$ & $i c_{2}$ & $i c_{6}^{5}$ & $i c_{6}$ & $3 i u_{2}^{\prime}$ \\
\hline \multirow[t]{11}{*}{$K_{1}$} & $a$ & $P_{1}^{(1)}$ & 2 & -1 & -1 & 0 & 0 & $\sqrt{3} i$ & $-\sqrt{3} i$ & 0 \\
\hline & & $P_{2}^{(1)}$ & 2 & -1 & -1 & 0 & 0 & $-\sqrt{3} i$ & $\sqrt{3} i$ & 0 \\
\hline & & $P_{3}^{(1)}$ & 2 & 2 & 2 & 0 & 0 & 0 & 0 & 0 \\
\hline & $b$ & $\left(\Gamma^{\prime}\right)_{1}^{(1)}=\Gamma_{7} E_{1}^{\prime}$ & 2 & 1 & -1 & 0 & 0 & $\sqrt{3}$ & $-\sqrt{3}$ & 0 \\
\hline & & $\left(\Gamma^{\prime}\right)_{2}^{(1)}=\Gamma_{8} E_{2}^{\prime}$ & 2 & 1 & -1 & 0 & 0 & $-\sqrt{3}$ & $\sqrt{3}$ & 0 \\
\hline & & $\left(\Gamma^{\prime}\right)_{3}^{(1)}=\Gamma_{9} E_{3}^{\prime}$ & 2 & -2 & 2 & 0 & 0 & 0 & 0 & 0 \\
\hline & & $u_{2}(r)$ & 1 & -1 & 1 & $i$ & $i$ & $-i$ & $-i$ & -1 \\
\hline & $c$ & $H_{1}^{(1)}$ & 2 & -1 & -1 & 0 & 0 & $-\sqrt{3}$ & $\sqrt{3}$ & 0 \\
\hline & & $H_{2}^{(1)}$ & 2 & -1 & -1 & 0 & 0 & $\sqrt{3}$ & $-\sqrt{3}$ & 0 \\
\hline & & $H_{3}^{(1)}$ & 2 & 2 & 2 & 0 & 0 & 0 & 0 & 0 \\
\hline & & $u_{1, H}(r)$ & 1 & 1 & 1 & 1 & $i$ & $i$ & $i$ & $i$ \\
\hline
\end{tabular}

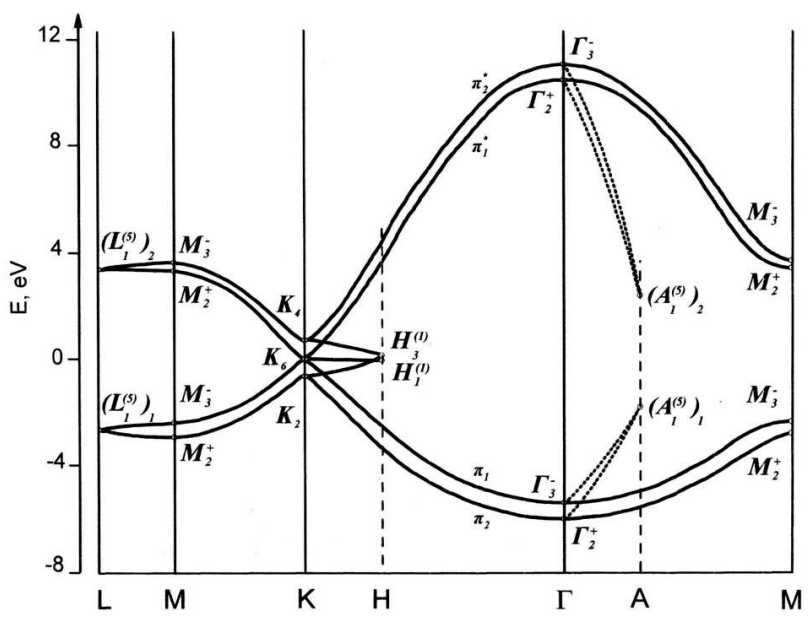

Fig. 6. Dispersion of the electron energy $\pi$-bands in the graphite crystals (letters mark the points in the Brillouin zone, and indexed letters do the irreducible projective representations of the corresponding projective classes)

tions are the sets of coefficients that are determined by the functions $u_{2}(r)$, i.e. such sets of coefficients for reducing factor-systems to standard forms, which correspond to the parameter $\alpha=-1$.

\subsection{Points $M$}

The factor-groups of the wave-vector groups with respect to the infinite translation-invariant subgroups at points $M$ of the single-layer graphene and crystalline graphite structures are isomorphic to the point group $m m m\left(D_{2 h}\right)$. The latter is a point symmetry group of equivalent directions for points $M$. The stars of the wave-vector groups at points $M$ in the both structures contain three vectors: in single-layer graphene $C_{L 1}$, these are $\left(\mathbf{k}_{M}\right)_{1}=-(1 / 2) \mathbf{b}_{2},\left(\mathbf{k}_{M}\right)_{2}=$ $=(1 / 2) \mathbf{b}_{1}$, and $\left(\mathbf{k}_{M}\right)_{3}=-(1 / 2)\left(\mathbf{b}_{1}-\mathbf{b}_{2}\right)$; for crystalline graphite $\gamma-C$, these are $\left(\mathbf{k}_{M}\right)_{1}=-(1 / 2) \mathbf{b}_{3}$, $\left(\mathbf{k}_{M}\right)_{2}=(1 / 2) \mathbf{b}_{2}$, and $\left(\mathbf{k}_{M}\right)_{3}=-(1 / 2)\left(\mathbf{b}_{2}-\mathbf{b}_{3}\right)$. The characters of projective representations $M_{\text {eq }}$, which belong to the class $K_{0}$ for both structures, are given in Table 11. The distributions $M_{\mathrm{vibr}}$ and $M_{\pi}$ over irreducible representations of the group $m m m\left(D_{2 h}\right)$ are presented in Table 12. The construction of the characters for the irreducible representations of the group $m m m\left(D_{2 h}\right)$ does not cause any difficulty, if one follows the recording sequence for the symmetry elements of the subgroup $\mathrm{mmm}$, namely, as they are written in the group $6 / \mathrm{mmm}$, but every representation that is even with respect to the inversion is followed by the corresponding odd one.

\subsection{Point L}

The factor-group of the wave-vector group with respect to the translation-invariant subgroup at point $L$ of crystalline graphite is also isomorphic to the 
Table 17. Factor-systems $\boldsymbol{\omega}_{1, L}\left(\boldsymbol{r}_{2}, \boldsymbol{r}_{1}\right)$ for point $\boldsymbol{L}$ in the Brillouin zone of crystalline graphite (the spatial group $P 6_{3} / m m c\left(D_{6 h}^{4}\right)$, the point group $m m m\left(D_{2 h}\right)$ ) (a) and standard factor-system $\omega_{1, L}^{\prime}\left(r_{2}, r_{1}\right)$

corresponding to its standard form $(b)$. The lower part of Table 17, $a$ contains the values of the function $u_{1, L}(r)$ that transforms the factor-system $\omega_{1, L}\left(r_{2}, r_{1}\right)$ for point $L$ to the standard

form $\omega_{1, L}^{\prime}\left(r_{2}, r_{1}\right) \equiv \omega_{(5)}^{\prime}\left(r_{2}, r_{1}\right)$

\begin{tabular}{|c|c|c|c|c|c|c|c|c|c|}
\hline$\omega_{1, L}\left(r_{2}, r_{1}\right)$ & i) $r_{2}$ & $\begin{array}{l}1 \\
e\end{array}$ & $\begin{array}{c}2 \\
\left(u_{2}\right)_{1}\end{array}$ & $\begin{array}{c}3 \\
c_{2}\end{array}$ & $\begin{array}{c}4 \\
\left(u_{2}^{\prime}\right)_{1}\end{array}$ & $\begin{array}{l}5 \\
i\end{array}$ & $\begin{array}{c}6 \\
i\left(u_{2}\right)_{1}\end{array}$ & $\begin{array}{c}7 \\
i c_{2}\end{array}$ & $\begin{array}{c}8 \\
i\left(u_{2}^{\prime}\right)_{1}\end{array}$ \\
\hline 1 & $e$ & $1_{(1)}$ & $1_{(2)}$ & $1_{(3)}$ & $1_{(4)}$ & $1_{(5)}$ & $1_{(6)}$ & $1_{(7)}$ & $1_{(8)}$ \\
\hline 2 & $\left(u_{2}\right)_{1}$ & $1_{(2)}$ & $1_{(1)}$ & $-1_{(4)}$ & $-I_{(3)}$ & $1_{(6)}$ & $1_{(5)}$ & $-\mathbf{l}_{(8)}$ & $-1_{(7)}$ \\
\hline 3 & $c_{2}$ & $I_{(3)}$ & $1_{(4)}$ & $1_{(1)}$ & $1_{(2)}$ & $1_{(7)}$ & $\mathbf{I}_{(8)}$ & $\mathbf{l}_{(5)}$ & $1_{(6)}$ \\
\hline 4 & $\left(u_{2}^{\prime}\right)_{1}$ & $1_{(4)}$ & $1_{(3)}$ & $-1_{(2)}$ & $-1_{(1)}$ & $1_{(8)}$ & $1_{(7)}$ & $-1_{(6)}$ & $-1_{(5)}$ \\
\hline 5 & $i$ & $1_{(5)}$ & $\mathbf{I}_{(6)}$ & $-1_{(7)}$ & $-1_{(8)}$ & $1_{(1)}$ & $1_{(2)}$ & $-1_{(3)}$ & $-1_{(4)}$ \\
\hline 6 & $i\left(u_{2}\right)_{1}$ & $1_{(6)}$ & $1_{(5)}$ & $1_{(8)}$ & $1_{(7)}$ & $I_{(2)}$ & $1_{(1)}$ & $1_{(4)}$ & $1_{(3)}$ \\
\hline 7 & $i c_{2}$ & $1_{(7)}$ & $1_{(8)}$ & $-1_{(5)}$ & $-1_{(6)}$ & $I_{(3)}$ & $I_{(4)}$ & $-1_{(1)}$ & $-1_{(2)}$ \\
\hline & $i\left(u_{2}^{\prime}\right)_{1}$ & $1_{(8)}$ & $1_{(7)}$ & $1_{(6)}$ & $1_{(5)}$ & $1_{(4)}$ & $1_{(3)}$ & $1_{(2)}$ & $1_{(1)}$ \\
\hline & $u_{1, L}(r)$ & 1 & 1 & 1 & 1 & 1 & 1 & -1 & -1 \\
\hline
\end{tabular}

$a$

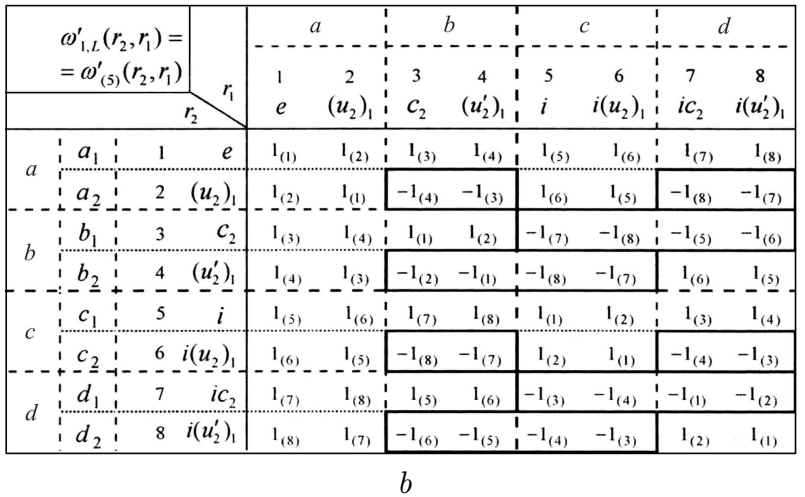

point group $m m m\left(D_{2 h}\right)$. The wave-vector star of point $L$ for the graphite $\gamma-C$ structure also contains three vectors: $\left(\mathbf{k}_{L}\right)_{1}=-(1 / 2)\left(\mathbf{b}_{1}+\mathbf{b}_{3}\right),\left(\mathbf{k}_{L}\right)_{2}=$ $=-(1 / 2)\left(\mathbf{b}_{1}-\mathbf{b}_{2}\right)$, and $\left(\mathbf{k}_{L}\right)_{3}=-(1 / 2)\left(\mathbf{b}_{1}+\mathbf{b}_{2}-\mathbf{b}_{3}\right)$. Table 17 demonstrates the factor-system $\omega_{1, L}\left(r_{2}, r_{1}\right)$ calculated by formula (8). With the help of the function values on the group $u_{1, L}(r)$ (they are indicated in the lower part of Table 17, a), it is reduced to the standard form $\omega_{1, L}^{\prime}\left(r_{2}, r_{1}\right)$, which is exhibited in Table $17, b$. The factor-systems $\omega_{1, L}\left(r_{2}, r_{1}\right)$ and $\omega_{1, L}^{\prime}\left(r_{2}, r_{1}\right)$ - in accordance with the values of the coefficients $\alpha, \beta$, and $\gamma$, which are determined from the factor-systems themselves - belong to the projective class $K_{5}$, i.e. $\omega_{1, L}^{\prime}\left(r_{2}, r_{1}\right) \equiv \omega_{(5)}^{\prime}\left(r_{2}, r_{1}\right)$. The characters of irreducible projective representations for point $L$ can easily be obtained if one knows the values of the function $u_{1, L}(r)$ and the characters of irreducible projective representations of the class $K_{5}$ corresponding to the standard factor-system of the class $K_{5}$, which are presented in Table 8 [6]. The irreducible projective representations for point $L$ can easily be determined from Table 9. The character of the projective equivalence representation at point $L$, i.e. the representation $L_{\mathrm{eq}}$, is given in Table 11. The distribution of representations $L_{\mathrm{vibr}}$ and $L_{\pi}$ over irreducible projective representations of the class $K_{5}$ of the group $\mathrm{mmm}$ is shown in Table 12.

Figure 6 schematically illustrates the dispersion of the electron energy $\pi$-bands in graphite crystals (the letters mark the points in the Brillouin zone, and the indexed letters do the irreducible projective representations of the corresponding projective classes). The dispersion of the electron $\pi$-bands is schematically presented in Fig. 6 for all high-symmetry points in the Brillouin zone of graphite crystals. Our results have a good qualitative agreement with the results of numerical calculations carried out in works $[14,15]$. At the same time, our results also include the qualitative behavior of the dispersion of electron bands along the high-symmetry line $\Gamma-\Delta-A$. Furthermore, the symbolic notations of representations that correspond to the classification of energy states by the irreducible projective representations of certain projective classes for various points in the Brillouin zone, which was made in this work for the first time, correspond to the exact values of characters and, hence, the matrices of irreducible projective representations, which exactly describe the symmetry of vibrational and electron excitations.

\section{Conclusions}

1. A method has been developed for constructing the correct multiplication tables of spinors, the wave functions of quantum states with a half-integer spin. In effect, this is a method to calculate the factorsystems of the projective class $K_{1}$, including a procedure of their reduction to the standard form.

2 . The standard factor systems and their corresponding irreducible representations for all projective classes of the hexagonal symmetry group $6 / \mathrm{mmm}\left(D_{6 h}\right)$ and its subgroups have been constructed for the first time. 
3. The classes of projective representations for various points in the Brillouin zone of crystalline graphite have been identified for the first time. The characters of irreducible projective representations are constructed for each of them. The symmetric distribution over the irreducible projective representations of vibrational excitations and electron states that are determined by the structure of the $\pi$-bands is determined.

4. A symmetric theoretical-group description of the dispersion of vibrational and electron excitations in crystalline graphite in view of the changes in the projective classes is made for the first time for various points in the Brillouin zone. Their correlations with vibrational and electron excitations in singlelayer graphene are revealed.

1. O.V. Kovalev. Irreducible Representations of the Space Groups (Gordon and Breach, 1965).

2. O.V. Kovalev. Irreducible and Induced Representations and Co-Representations of Fedorov groups (Nauka, 1986) (in Russian).

3. J.D. Bernal. The structure of graphite. Proc. Roy. Soc. London A 106, 749-773 (1924).

4. C. Herring. Effect on time-reversal symmetry on energy band of crystals. Phys. Rev. 52, 361 (1937).

5. E.A. Wood. The 80 diperiodic groups in three dimensions. Bell System Tech. J. 43, 541 (1964).

6. G.L. Bir, G.E. Pikus, Symmetry and Strain-Induced Effects in Semiconductors (Wiley, 1974).

7. H.A. Bethe. Termaufspaltung in Kristallen. Ann. Physik 395, 133 (1929).

8. V.O. Gubanov, L.N. Ovander. Development of the Bethe method for the construction of two-valued space group representations and two-valued projective representations of point groups. Ukr. J. Phys. 60 (9), 950 (2015).

9. E.P.Wigner. Group Theory and Its Application to the Quantum Mechanics of Atomic Spectra (Academic Press, 1959).

10. D.S. Balchuk, M.M. Bilyi, V.P. Gryshchuk, V.O. Gubanov, V.K. Kononov. Symmetry of vibrational modes, timeinversion invariance of energy states, and Raman light scattering in $4 \mathrm{H}-$ and $6 \mathrm{H}-\mathrm{SiC}$ crystals. 1. Classification of energy states in Brillouin zones. Ukr. Fiz. Zh. 41, 146 (1996) (in Ukrainian).

11. T.L. Botte, V.A. Gubanov, L.A. Komarova, N.M. Belyi, S.V. Kovrigin, I.A. Petrusha, A.A. Shulzhenko. Correla- tion of vibrational modes and dispersion of phonon excitations in layered crystals of graphite and graphite-like boron nitride. 1. Classification and correlation of phonon states with zero quasimomentum. Nanosist. Nanomater. Nanotekhnol. 5, 287 (2007) (in Russian).

12. V.A. Gubanov, L.A. Komarova, N.M. Belyi, S.V. Kovrigin. Correlation of vibrational modes and dispersion of phonon excitations in layered crystals of graphite and graphite-like boron nitride. 2. Dispersion of phonon states with nonzero quasi-momentum and the width of phonon bands. Nanosist. Nanomater. Nanotekhnol. 5, 307 (2007) (in Russian).

13. M.S. Dresselhaus, G. Dresselhaus, A. Jorio. Group Theory. Application to the Physics of Condensed Matter (Springer, 2008).

14. E. Doni, G. Pastori Parravicini. Energy bands and optical properties of hexagonal boron nitride and graphite. Nuovo Cimento B 64, 117 (1969).

15. F. Bassani, G. Pastori Parravicini. Electronic States and Optical Transitions in Solids (Pergamon Press, 1975).

Received 29.01.18.

Translated from Ukrainian by O.I. Voitenko

В.О. Губанов, А.П. Науменко,

М.М. Білий, І.С. Доченко, О.М. Навозенко,

М.М. Сабов, Л.А. Булавін

КОРЕЛЯЦІЯ ЕНЕРГЕТИЧНИХ СПЕКТРІВ КОЛИВАЛЬНИХ І ЕЛЕКТРОННИХ ЗБУДЖЕНЬ ТА ЇХНЯ ДИСПЕРСІЯ В ГРАФІТІ ТА ГРАФЕНІ

Р е з ю м е

Досліджено кореляцію коливальних мод, електронних збуджень та їх дисперсійні залежності в одношаровому графені та кристалах графіту. Для інтерпретації таких кореляцій вперше використано методи теорії проективних представлень точкових та просторових груп симетрії. Визначено кореляції енергетичних спектрів коливальних та електронних збуджень і умови сумісності незвідних проективних представлень в описах квантових станів графену і кристалічного графіту для різних точок їх зон Бріллюена. Для проективних представлень всіх проективних класів гексагональної системи вперше побудовано стандартні фактор-системи, в тому числі вперше визначено фактор-системи для електронних станів, за допомогою яких вперше знайдено коректні таблиці множення спінорів, тобто таблиці множення елементів в подвійних групах симетрії. На основі зазначеного вище, надано розподіли за типами симетрії коливальних збуджень для всіх точок високої симетрії зон Бріллюена одношарового графену і кристалічного графіту. 